\title{
Multi-multifractal decomposition of digraph recursive fractals
}

\section{Dominique Simpelaere}

\begin{abstract}
In many situations, both deterministic and probabilistic, one is interested in measure theory in local behaviours, for example in local dimensions, local entropies or local Lyapunov exponents. It has been relevant to study dynamical systems, since the study of multifractal can be further developped for a large class of measures invariant under some map, particularly when there exist strange attractors or repelers (hyperbolic case). Multifractal refers to a notion of size, which emphasizes the local variations of the weight of a measure, of the entropy or the Lyapunov exponents. All these notions are explicited in the case of digraph recursive fractal studied by Edgar \& Mauldin where some questions are given. We study the extremal measures and introduce the notion of multi-multifractality which may be useful in problems of rigidity.
\end{abstract}

\section{Introduction.}

In many situations implicated the dimension of measures, singular measures are investigated, and more precisely how densely the singularities of a measure are distributed.

Let $(X, d)$ be a compact metric space and $\mu$ be a Borel probability measure. The decay rates of the measure $\mu$ of small balls are determined in order to define local dimensions. The singularities of the measure $\mu$ 
are specified by

$$
\underline{d}_{\mu}(x)=\underline{\lim }_{r \rightarrow 0} \frac{\ln \mu(B(x, r))}{\ln r} \quad \text { and } \quad \bar{d}_{\mu}(x)=\varlimsup_{r \rightarrow 0} \frac{\ln \mu(B(x, r))}{\ln r},
$$

and when $\underline{d}_{\mu}(x)=\bar{d}_{\mu}(x)=d_{\mu}(x)$, the measure $\mu$ has pointwise dimension $d_{\mu}(x)$, and it is said that $\mu$ is exact dimensional [Si1], [Y] if for $\mu$ almost every point $x$ we have $d_{\mu}(x)=d_{\mu}=$ constant.

Even for nice measures, it is not expected that this pointwise dimension exists or the measure $\mu$ to be exact dimensional [LM], [S]. The singularity sets are then defined for any real number $\alpha \geq 0$ by

$$
\begin{aligned}
& C_{\alpha}^{-}=\left\{x \in X: \underline{d}_{\mu}(x)=\alpha\right\}, \\
& C_{\alpha}^{+}=\left\{x \in X: \bar{d}_{\mu}(x)=\alpha\right\}, \\
& C_{\alpha}=C_{\alpha}^{+} \cap C_{\alpha}^{-},
\end{aligned}
$$

which is called the multifractal decomposition.

This concept first appeared in a paper of physicists [HJKPS] where it was suggested to study the so-called dimension spectrum $f(\alpha)$, i.e.

$$
f(\alpha)=\operatorname{HD}\left(C_{\alpha}\right) \quad \text { and } \quad f(\alpha) \equiv-\infty, \quad \text { if } C_{\alpha}=\varnothing .
$$

There exist many definitions of dimension [F2], [P2]: Packing-dimension, Box-dimension... For theoretical purposes the Hausdorff dimension is prefered: for any Borel set $A$ and any positive real number $\tau$, put

$$
\operatorname{HD}_{\tau, \varepsilon}(A)=\inf _{\substack{A \subset \cup A_{i} \\\left|A_{i}\right|<\varepsilon}}\left\{\sum_{i \geq 0}\left|A_{i}\right|^{\tau}\right\}
$$

and

$$
\operatorname{HD}_{\tau}(A)=\lim _{\varepsilon \rightarrow 0} \operatorname{HD}_{\tau, \varepsilon}(A) \in[0,+\infty] .
$$

We obtain finally the Hausdorff dimension (which derives from a measure) by the following

$$
\operatorname{HD}(A)=\sup \left\{\tau: \operatorname{HD}_{\tau}(A)=+\infty\right\}=\inf \left\{\tau: \operatorname{HD}_{\tau}(A)=0\right\},
$$

and the Hausdorff measure of $A$ is the value $\operatorname{HD}_{\mathrm{HD}(A)}(A) \in[0,+\infty]$.

We define the dimension of a Borel measure $\mu$ by

$$
\operatorname{HD}(\mu)=\inf \{\operatorname{HD}(A): A \text { a Borel set and } \mu(A)=1\} .
$$


In fact it has been found relevant information in a large class of measures, namely dynamical systems $(X, \mu, T)$ where the map $T: X \hookleftarrow$ is ergodic and the measure $\mu$ is $T$-invariant. The first rigorous result [CLP] was the multifractal analysis of $C^{2}$ one-dimensional Markov maps. Many articles appeared on this subject: $[R]$ for Cookie-cutters, [Lo] for hyperbolic Julia sets, [Si1] for Axiom A surface diffeomorphisms. Other models have been developped: multiplicative chaos (tree structure) which is a model of the phase transition of a system with random interactions in physics and chemistry, in polymers, turbulence, thermodynamics, rainfall distribution - random measures with fixed supports $[\mathrm{HW}]$ or with random supports [F1]; iterated function systems [BPS1], [BPS2], [BMP], [CM], [CLP], [EM], [K], [Lo], [O1], [O2], [Si1]. There are now many references that may be found in particular in [P2], especially in the very well-known case of self-similarity for sets or measures $[\mathrm{Mo}],[\mathrm{MR}]$.

One physical motivation is when ergodic-time averages along the process converge to a measure $\mu:=\lim _{n \rightarrow+\infty}(1 / n) \sum_{i=0}^{n-1} \delta_{T^{i}(x)}$ which describes the occupation of the attractor under iterations of $T$. This measure $\mu$ is the one that can be seen on the screen when computing the iterates of a point under the dynamical system. This is the case for SBR (Sinaï-Bowen-Ruelle) measures of diffeomorphisms of smooth Riemannian manifolds which contain a compact hyperbolic attractor $\Lambda$ of $T$. The limit measure $\mu$ has absolutely continuous conditional measures on unstable manifolds [HY], and the measure $\mu$ describes the orbit distribution of points in a basin $B \supset \Lambda$. Clearly, one sees how densely the singularities of $\mu$ are distributied - areas are darker and darker when there are more and more visits.

Most of the measures in the literature are equilibrium measures Gibbs measures - and therefore are very common and typical in physics. In some cases explicit formulae can be obtained [BPS1], [BPS2], [R], [Si1], and in all the cases the dimension spectrum $f$ is proved to be real analytic.

A new approach is suggested when looking at the distribution along orbits. We define for any $x \in X$ and any integer $q \geq 2$ the quantity [GHP], [HP], [P1], [PT],

$$
\begin{array}{r}
C(x, q, r, n)=\frac{1}{n^{q}} \#\left\{\left(i_{1}, \ldots, i_{q}\right): d\left(T^{i_{j}}(x), T^{i_{k}}(x)\right)<r\right. \\
\text { for } \left.0 \leq i_{j}<i_{k}<n\right\} .
\end{array}
$$


If the measure $\mu$ is ergodic, we have for $\mu$ allmost every $x$,

$$
\lim _{n \rightarrow+\infty} C(x, q, r, n)=\int_{X} \mu(B(y, r))^{q-1} d \mu(y) .
$$

Provided the limit exists, we define the HP spectrum

$$
\begin{aligned}
&(1-q) C_{q}(x)=\lim _{r \rightarrow 0} \lim _{n \rightarrow+\infty} \frac{\ln C(x, q, r, n)}{\ln r} \\
& \stackrel{\mu \text { a.e. }}{=} \frac{\ln \left(\int_{X} \mu(B(y, r))^{q-1} d \mu(y)\right)}{\ln r} .
\end{aligned}
$$

In $[\mathrm{O} 1],[\mathrm{O} 2]$, [P1], [Si2] this function is generalized to real numbers and is called the correlation dimension,

$$
C(\beta)=\lim _{r \rightarrow 0} \frac{\ln \left(\int_{X} \mu(B(y, r))^{\beta} d \mu(y)\right)}{\ln r}, \quad \text { for all } \beta \in \mathbb{R},
$$

provided the limit exists, which is for $\beta=1$ the average of the singularities of $\mu$ [Si2].

This function can be seen in the following way (order two approach) suggested by D. Ruelle and described in [P1]. Consider the product metric space $Y=X \times X$ equipped with the metric

$$
d^{\prime}\left(\left(x_{1}, y_{1}\right),\left(x_{2}, y_{2}\right)\right)=d\left(x_{1}, y_{1}\right)+d\left(x_{2}, y_{2}\right)
$$

and define the direct product measure $\nu=\mu \times \mu$. Define the diagonal

$$
D=\{(x, x) \in Y\} \text { and for } r>0, D_{r}=\left\{y \in Y: d^{\prime}(y, D)<r\right\} .
$$

We then obtain

$$
\nu\left(D_{r}\right)=\int_{X} \mu(B(x, r)) \mu(d x),
$$

and therefore we have

$$
\frac{\ln \nu\left(D_{r}\right)}{\ln r}=\frac{\ln \int_{X} \mu(B(x, r)) \mu(d x)}{\ln r} \underset{r \rightarrow 0}{\rightarrow} C(1) .
$$

This function $C$ plays an important role in the numerical investigation of some models and the procedure is simple and runs fast [GHP], [P1]. 
In multifractal analysis there are two methods: the first one comes from the theory of operators (Perron-Froenius) and gives the existence, uniqueness and regularity of the solution [EM]. The other one is based on large deviations and thermodynamics, and leads to explicit formulae [CLP], [Si1]. The latter is described in the following.

Using large deviations and under suitable assumptions, we have the multifractal formalism, i.e. the dimension spectrum $f$ is the LegendreFenchel transform of a function $F$, called free energy function, concave and at least $C^{1}$, i.e.

$$
f(\alpha)=\inf _{t \in \mathbb{R}}\{t \alpha-F(t)\}
$$

where $F$ is derived from a sequence of partition functions $\left\{Z_{n}\right\}_{n \geq 1}$

(6) $F(\beta)=\lim _{n \rightarrow+\infty}-\frac{1}{n} \ln _{b(\beta, n)} Z_{n}(\beta) \quad\left(:=F_{n}(\beta)\right), \quad$ for all $\beta \in \mathbb{R}$.

These partition functions are defined by the following

$$
Z_{n}(\beta)=\sum_{\substack{U \in Q_{n} \\ \mu(U)>0}} \mu(U)^{\beta}, \quad \text { for all } \beta \in \mathbb{R}
$$

where $\left\{Q_{n}\right\}_{n \geq 1}$ is a well chosen sequence of partitions (typically the Markov partition $\left\{\mathcal{P}_{n}\right\}_{n \geq 1}$ generated by the dynamics and the iterates under $T$ [Bo], [Ru]) whose diameters tend to 0 when $n$ goes to $+\infty$ (for $b(n(\beta))$ see $(31)$ and $(32))$.

There is another intrinsic free energy function [CLP], [RU], [Si1] associated to the Markov partition $\left\{\mathcal{P}_{n}\right\}_{n \geq 1}$ defined on $\mathbb{R}^{2}$ by (see Theorem A and (35))

$$
G_{\mathcal{D}}(x, y)=\lim _{n \rightarrow+\infty} \frac{1}{n} \ln G_{\mathcal{D}}^{(n)}(x, y), \quad \text { for all }(x, y) \in \mathbb{R}^{2}
$$

with

$$
G_{\mathcal{D}}^{(n)}(x, y)=\sum_{A \in \mathcal{P}_{n}} \mu(A)^{x}|A|^{y}, \quad \text { for all }(x, y) \in \mathbb{R}^{2}
$$

For these thermodynamic quantities it is proved that [O1], [O2], [Si2]

$$
C(\beta)=F(\beta)+1, \quad \text { for all } \beta \in \mathbb{R},
$$


and this equality holds if and only if $F$ can be associated to a sequence of uniform partitions. It is also proved that [CLP], [Si2]

$$
\mathrm{HD}(\mu)=\inf \{\operatorname{HD}(A): A \text { a Borel set and } \mu(A)=1\}=d_{\mu}=F^{\prime}(1) .
$$

The main result in multifractal analysis is the following: $f$ is smooth (real analytic or $C^{\infty}$ ) and strictly concave on an interval $] \alpha_{\min }, \alpha_{\min }[\subset$ $\mathbb{R}^{+*}$ and is the Legendre-Fenchel transform of a function $F$ of same regularity, except in the degenerate case where it is defined at one point (this case can be described).

There exist also multifractal decompositions for (Kolmogorov-Sinaï) entropy and Lyapunov exponents - decompositions into level sets.

For the entropy spectrum, let $\{\xi\}$ be a generating partition, i.e. if $\mathcal{B}(X)$ is the Borel algebra, then $\mathcal{B}(X)=\bigvee_{i \geq 0} T^{-i}(\xi) \mu \bmod 0$ (for example the Markov partition) and $\xi_{n}(x)$ be the element of the partition $\xi_{n}$ at rank $n$,

$$
\xi_{n}=\bigvee_{i=0}^{n-1} T^{-i}(\xi),
$$

which contains the point $x$. Then define local entropy,

$$
h_{\mu}(x)=h_{\mu}(x, \xi, T)=\lim _{n \rightarrow+\infty}-\frac{1}{n} \ln \mu\left(\xi_{n}(x)\right),
$$

provided the limit exists (it exists for $\mu$ almost every point $x$ in the ergodic case), and for $\mu$ almost every point $x, h_{\mu}(x)=h_{\mu}$ ( $\mu$ is exact for the entropy in the ergodic case), the entropy of the dynamical system (the exact value).

We define the level sets for entropy for any real $\eta \geq 0$ by

$$
E(\eta)=\left\{x: h_{\mu}(x)=\eta\right\} \quad \text { and } \quad E_{n}(\eta)=\operatorname{HD}(E(\eta)),
$$

which is the entropy spectrum.

For the local Lyapunov exponent, let $M$ be a smooth manifold, $T: M \hookleftarrow$ a $C^{2}$ conformal expanding map leaving invariant a compact subset $\Lambda$ of $M$. Let $\mu$ be a $T$-invariant probability measure on $\Lambda$. We have for any tangent vector $\vec{v} \in T_{x}(\Lambda)$,

$$
\chi_{\mu}(x)=\lim _{n \rightarrow+\infty} \frac{1}{n} \ln \left\|d T_{x}^{n}(\vec{v})\right\|,
$$

provided the limit exists (it exists $\mu$ almost everywhere), and for $\mu$ alomost every point $x, \chi(x)=\chi_{\mu}$, the Lyapunov exponent of the dynamical system (the exact value). 
We define the level sets for Lyapunov exponents: for any real $\vartheta \geq 0$, consider

$$
L(\vartheta)=\left\{x: \chi_{\mu}(x)=\vartheta\right\} \quad \text { and } \quad L_{y}(\vartheta)=\operatorname{HD}(L(\vartheta))
$$

which is the Lyapunov spectrum.

We then have the following multifractal decompositions

$$
\left\{\begin{aligned}
\Lambda= & \left\{x: h_{\mu}(x) \text { does not exist }\right\} \cup\left\{x: h_{\mu}(x)=h_{\mu}\right\} \\
& \bigcup_{\alpha \neq h_{\mu}}\left\{x: h_{\mu}(x)=\alpha\right\}, \\
\Lambda= & \left\{x: \chi_{\mu}(x) \text { does not exist }\right\} \cup\left\{x: \chi_{\mu}(x)=\chi_{\mu}\right\} \\
& \bigcup_{\alpha \neq \chi_{\mu}}\left\{x: \chi_{\mu}(x)=\alpha\right\},
\end{aligned}\right.
$$

and the corresponding spectra. Notice that the existence of the exact values for the different spectra are given by: the Eckmann-Ruelle conjecture [BPS1] for dimension, the Shannon-McMillan-Breiman theorem for entropy and the Kingman theorem for Lyapunov exponents.

Notice that in general we have

$$
\operatorname{HD}\left(\left\{x \in X: h_{\mu}(x) \text { does not exist }\right\}\right)>0
$$

and similarly

$$
\operatorname{HD}\left(\left\{x \in X: \chi_{\mu}(x) \text { does not exist }\right\}\right)>0 \quad(=\operatorname{dim}(X)) .
$$

Our aim is to answer to questions found in [EM]: completness of the dimension spectrum (and finally the other spectra), problems at the bounds of the interval of definition of the spectra, case where the transition matrix is not irreducible...

Results found in [EM] are given in Section 2. We find again these results and generalize them in a different framework (Section 3). Then using notations and results of Section 3, let us define the following.

In the case of expanding Markov maps, a map $T \in C^{1+\delta}(\Lambda)$ is given, and for $x \in \Lambda, J(x)=-\ln T^{\prime}(x)<0\left(\in C^{\delta}(\Lambda)\right)$. The $T$ invariant measure $\mu$ is a Gibbs measure associated to the potential $\xi \in C^{\delta}(\Lambda)<0$. Since the set $\Lambda$ is compact the functions $\xi$ and $J$ take their values in compacts sets $[a, b]$ and $[c, d]$ since there are continuous.

For any real number $\beta$ we define a Gibbs measure $\mu_{\beta}$ associated to the potential $\xi_{\beta}=\beta \xi-F(\beta) J$ (and $\mu_{ \pm \infty}$ are limits when $\beta \rightarrow \pm \infty$ ). 
Consider

$$
\alpha_{\min }=\frac{\int_{\Lambda} \xi d \mu_{+\infty}}{\int_{\Lambda} J d \mu_{+\infty}}=\alpha_{+\infty} \quad \text { and } \quad \alpha_{\max }=\frac{\int_{\Lambda} \xi d \mu_{-\infty}}{\int_{\Lambda} J d \mu_{-\infty}}=\alpha_{-\infty} .
$$

We then have the following results.

Theorem A. For any $(\beta, s, t) \in \mathbb{R}^{3}$ we have

$$
\begin{gathered}
G(s, t)=P(s \xi+t J), \\
F(\beta)=\frac{h_{\mu_{\beta}}+\beta \int_{\Lambda} \xi d_{\mu_{\beta}}}{\int_{\Lambda} J d_{\mu_{\beta}}}, \\
G(\beta,-F(\beta))=0,
\end{gathered}
$$

and

$$
F(\beta)=-\psi(\beta)
$$

In the degenerate case the different spectra are reduced to points. Otherwise we can associate a family of probability measures $\left\{\mu_{\beta}\right\}_{\beta \in \overline{\mathbb{R}}}$, and we have the following.

Theorem B. We have in the general case $+\infty$.

- $C_{\alpha} \neq \varnothing$ if and only if $\alpha \in\left[\alpha_{\min }, \alpha_{\max }\right]$ where $0<\alpha_{\min }<\alpha_{\max }<$

- For all $\alpha \in\left[\alpha_{\min }, \alpha_{\max }\right]$ there exists a unique $\beta=f^{\prime}(\alpha) \in \overline{\mathbb{R}}$ such that $\mu_{\beta}$ is exact dimensional, and

$$
f(\alpha)=\operatorname{HD}\left(C_{\alpha}\right)=\operatorname{HD}\left(\mu_{\beta}\right)=d_{\mu_{\beta}}=\frac{\int_{\Lambda}-\xi d \mu_{\beta}}{\int_{\Lambda}-J d \mu_{\beta}}=\frac{h_{\mu_{\beta}}(T)}{\chi_{\mu_{\beta}}(T)} .
$$

- $\mu$ is exact dimensional: $\operatorname{HD}(\mu)=d_{\mu}=f(\alpha(1))$ where $\alpha(1)=$ $F^{\prime}(1)$. 
Theorem C. We have in the general case:

1) For the entropy spectrum (9) and (10): $+\infty$.

- $E(\eta) \neq \varnothing$ if and only if $\eta \in\left[\eta_{\min }, \eta_{\max }\right]$ where $0<\eta_{\min }<\eta_{\max }<$

- For all $\eta \in\left[\eta_{\min }, \eta_{\max }\right]$ there exists a unique $\beta \in \overline{\mathbb{R}}$ such that $\eta=\int_{\Lambda}-\xi d \mu_{\beta}=h_{\mu_{\beta}}\left(\mu_{\beta}\right.$ is exact dimensional $)$, and

$$
E_{n}(\eta)=\operatorname{HD}(E(\eta))=\operatorname{HD}\left(\mu_{\beta}\right)=d_{\mu_{\beta}}=\frac{\int_{\Lambda}-\xi d \mu_{\beta}}{\int_{\Lambda}-J d \mu_{\beta}}=\frac{h_{\mu_{\beta}}}{\chi_{\mu_{\beta}}}=f(\alpha)
$$

where $\alpha=F^{\prime}(\beta)$.

- $\mu$ is exact dimensional: for $\eta=h_{\mu}(\beta=1)$, we have $\mu(E(\eta))=1$ and

$$
E_{n}(\eta)=\operatorname{HD}\left(E\left(h_{\mu}\right)\right)=d_{\mu}=\frac{h_{\mu}}{\chi_{\mu}} .
$$

2) For the Lyapunov spectrum (11) and (12):

- $L(\vartheta) \neq \varnothing$ if and only if $\vartheta \in\left[\vartheta_{\min }, \vartheta_{\max }\right]$ where $0<\vartheta_{\min }<$ $\vartheta_{\max }<+\infty$.

- For all $\vartheta \in\left[\vartheta_{\min }, \vartheta_{\max }\right]$ there exists a unique $\beta \in \overline{\mathbb{R}}$ such that $\vartheta=\int_{\Lambda}-J d \mu_{\beta}=\chi_{\mu_{\beta}}\left(\mu_{\beta}\right.$ is exact dimensional $)$, and

$$
L_{y}(\vartheta)=\operatorname{HD}(L(\vartheta))=\operatorname{HD}\left(\mu_{\beta}\right)=d_{\mu_{\beta}}=\frac{\int_{\Lambda}-\xi d \mu_{\beta}}{\int_{\Lambda}-J d \mu_{\beta}}=\frac{h_{\mu_{\beta}}}{\chi_{\mu_{\beta}}}=f(\alpha),
$$

where $\alpha=F^{\prime}(\beta)$.

and

- $\mu$ is exact dimensional: for $\vartheta=\chi_{\mu}(\beta=1)$, we have $\mu(L(\vartheta))=1$

$$
L_{y}(\vartheta)=\operatorname{HD}\left(L\left(\chi_{\mu}\right)\right)=d_{\mu}=\frac{h_{\mu}}{\chi_{\mu}} .
$$

Theorem D. The extremal measures $\mu_{ \pm \infty}$ are uniform on their Cantor-like fractal supports. 
In Section 2 we define the model and the results (theorems 1 and 2) obtained in $[\mathrm{EM}]$.

In Section 3 we give a short exposition concerning the thermodynamic formalism that we use for our computations in the next sections.

In Section 4 we find again and generalize the results in [EM] by proving theorems $\mathrm{A}$ and $\mathrm{B}$.

Section 5 deals with the multifractal spectra, entropy and Lyapunov exponents, which correspond to the level sets (10) and (12), and we prove Theorem $\mathrm{C}$.

In Section 6 we develop a new concept: multi-multifractality, which allows us to give answers concerning extremal points (the points $\alpha_{ \pm \infty}$ ) in a quite simple fashion and we prove Theorem D. In particular we give some graphs of the functions we have studied.

Section 7 is devoted to discussion and new questions.

\section{The model and the operator theory.}

We start from a directed multigraph $(V, E)[\mathrm{EM}]$. The set $E=$ $\left\{e_{1}, \ldots, e_{k}\right\}$ consists of the edges of the graph, and the elements of $V=$ $\{u, v, \ldots, w\}$ are the vertices. This graph is supposed to be strongly connected, that means there is a path from any vertex to any other along the edges of the path (if not we decompose it into connex components).

Now we define notions of length and measure (mass) in order to compute local dimensions (1).

A path of length $k$ in the graph is a finite string

$$
\gamma=e_{1} e_{2} \cdots e_{k}
$$

of edges, and to each edge $e$ correspond a ratio $r(e) \in] 0,1[$ (parameter of a homethety in $\left.\mathbb{R}^{n}\right)$, and $r(\gamma)=r\left(e_{1}\right) r\left(e_{2}\right) \cdots r\left(e_{k}\right)$. The subset $E_{u v}$, the edges from $u$ to $v$, is a partition of $E$ for $(u, v) \in V^{2}$. The set $E_{u v}^{(k)}$ is composed of all the paths of length $k$ that start at $u$ and end at $v$, $E_{u}^{(k)}$ is the set of paths of length $k$ starting at $u$, and $E_{u}$ is the set of infinite paths starting at $u$.

For any vertex $u \in V$, let $J_{u}$ be a nonempty compact subset of $\mathbb{R}^{n}$. Actually we may assume for simplicity that the diameter of the set $\left|J_{u}\right|=1$ for any $u \in E$.

A digraph recursive fractal, based on seed set $J_{u}$ and ratios $r(e)$, 
is the set

$$
K_{u}=\bigcap_{k \geq 0}\left(\bigcup_{\gamma \in E_{u}^{(k)}} J(\gamma)\right)
$$

where the sets $J(\gamma)$ are choosen recursively:

i) $J\left(\Lambda_{u}\right)=J_{u}$ where $\Lambda_{u}$ is the empty path from $u$ to $u$.

ii) For $\gamma$ of length $k$ with terminal vertex $v$, the set $J(\gamma)$ is geometrically similar to $J_{v}$ with reduction ratio $r(\gamma)$.

iii) For $\gamma$ of length $k$ with terminal vertex $v$, the sets $J(\gamma e), e \in$ $E_{v}$, are nonoverlapping subset of $J(\gamma)$ (they intersect at most at their boundaries: "open set condition").

There are many choices to place the sets $J(\gamma e)$ in $J(\gamma)$, and for example consider the "self-similar graph" fractals using similarities $H_{e}$ : $\mathbb{R}^{n} \longrightarrow \mathbb{R}^{n}$, one for each edge $e \in E$. Define for any $\gamma=e_{1} e_{2} \cdots e_{k} \in$ $E_{u v}^{(k)}$

$$
J(\gamma)=H_{e_{1}} H_{e_{2}} \cdots H_{e_{k}}\left(J_{v}\right),
$$

where the seed set $J_{v}$ must be choosen such that iii) is satisfied.

We now define the measure of Markov type $\mu_{u}$ on $K_{u}$ recursively: we start with $\mu_{u}\left(J_{u}\right)=1$, and the mass is distributed among the subsets $J(e), e \in E$, so that $J(e)$ has mass $p(e)$. Once the mass of a set $J(\gamma)$ has been assigned, then it is distributed among the subsets $J(\gamma e)$ according to the values of $p(e)$. With (14) we get finally a unique probability measure depending on the choice of the number $\left((p(e))_{e \in E}\right.$. As for the definition of $r(\gamma)$, we get $p(\gamma)=p\left(e_{1}\right) \cdots p\left(e_{k}\right)$ for $\gamma=e_{1} e_{2} \cdots e_{k}$.

It implies that $p$ is defined on "cylinders", and then by the Kolmogorov consistency theorem a unique measure $\mu_{u}$ on $K_{u}$ is defined.

Let for $(\sigma, k)=\left(e_{1} e_{2} \cdots e_{k}\right)$ the finite string of length $k$,

$$
\begin{aligned}
h_{u}: E_{u} & \longrightarrow K_{u} \\
\sigma & \longmapsto \bigcap_{k \geq 1} J\left(\sigma_{\mid k}\right)
\end{aligned}
$$

(representation of the coding sequences of the trajectories, one-to-one at least on a set of $\mu$ measure 1 - the points with more than two representations have no local dimension). We have $\mu=\nu_{u} \circ h_{u}^{-1}$ where $\nu_{u}$ is defined on $E_{u}$ (it is defined on the cylinders). 
Let $A$ be the transition matrix associated to the Markov partition given by the iterations of the sets $J_{v}, v \in E$, by the map $H$ which determines the distribution of the $J(v e), e \in E$, inside $J(v)$ (for example in the case of "self-similar graph fractals", $H$ is composed of similarities $H_{v}: \mathbb{R}^{n} \longrightarrow \mathbb{R}^{n}$, for each edge $v$ ).

Define the matrix $B$,

$$
B_{u v}(\beta, s)=\sum_{e \in E_{u v}} p(e)^{\beta} r(e)^{s}, \quad(\beta, s) \in \mathbb{R}^{2}
$$

(compare with $(35)$ and the function $G_{\mathcal{D}}^{(0)}(\beta, s)$ ), and let $\phi(\beta, s)$ be the spectral radius of $B$. By the Perron-Frobenius theory of nonnegative matrices, $\phi$ is real analytic in both variables, and given any real number $\beta$, there exists a unique real number $s=\psi(\beta)$ such that $\phi(\beta, \psi(\beta))=1$. We get in particular $\operatorname{HD}\left(K_{u}\right)=\psi(0)=d$ which is independant of $u$.

Here are the results obtained in [EM].

Theorem 1. The function $\psi$ is real analytic, strictly decreasing from $+\infty$ to $-\infty$ and convex.

Let for any real number $\beta$,

$$
\alpha=\psi^{\prime}(\beta)>0 \quad \text { and } \quad f=\beta \alpha+\psi(\beta),
$$

and for $\gamma=e_{1} e_{2} \cdots e_{k}$,

$$
\delta(\gamma)=\frac{\ln p(\gamma)}{\ln r(\gamma)}=\frac{\ln \left(p\left(e_{1}\right) p\left(e_{2}\right) \cdots p\left(e_{k}\right)\right)}{\ln \left(r\left(e_{1}\right) r\left(e_{2}\right) \cdots r\left(e_{k}\right)\right)}
$$

and $\alpha_{\min }=\inf \{\delta(\gamma): \gamma$ is a simple cycle $\}\left(\alpha_{\max }=\sup \right)$.

Let $\left\{x_{v}\right\}_{v \in V}$ be the Perron numbers and consider the pairs $\left(\lambda_{v}, \rho_{v}\right)_{v \in V}$. We have, for all $v \in V, x_{v}>0$ and for all $u \in V$,

$$
\sum_{v \in V} \sum_{e \in E_{u v}} r(e)^{d} x_{v}^{d}=x_{u}^{d}
$$

for all $u \in V$,

$$
\sum_{v \in V} \sum_{e \in E_{u v}} P(e)=1
$$

where $P(e)=\rho_{u}^{-1} p(e)^{\beta} r(e)^{\psi(\beta)} \rho_{v}$. The real numbers $\pi_{u}$ define a stationary distribution for the Markov chain: given $X_{k}=u$, the conditional probability that $X_{k+1}=v$ is $\sum_{e \in E_{u v}} P(e)$. 
These are the transition probabilities for some stationary measure on $E_{u}, \nu_{u}^{(\beta)}$, a measure of Markov type defined on the cylinders of $E_{u}$. With the map $h_{u}$ it corresponds to a measure $\mu_{u}^{(\beta)}$ on $K_{u}$,

$$
\nu_{u}^{(\beta)}(\gamma)=\rho_{u}^{-1} p(\gamma)^{\beta} r(\gamma)^{\psi(\beta)} \rho_{v} \quad \text { and } \quad \mu_{u}^{(\beta)}=\nu_{u}^{(\beta)} \circ h_{u}^{-1} .
$$

We then have defined for all $u \in V$ measures $\nu_{u}^{(\beta)}, \beta \in \mathbb{R}$, on the sets $E_{u}$ by its transition probabilities, and therefore measures $\mu_{u}^{(\beta)}, \beta \in \mathbb{R}$, on the sets $K_{u}, \mu_{u}^{(\beta)}=\nu_{u}^{(\beta)} \circ h_{u}^{-1}$.

Consider for any $u \in V$,

$$
\left\{\begin{array}{l}
K_{u}^{(\alpha)}=\left\{x \in K_{u}: \lim _{r \rightarrow 0} \frac{\ln \mu_{u}(B(x, r))}{\ln r}=\alpha\right\}, \\
E_{u}^{(\alpha)}=\left\{\sigma \in E_{u}: \lim _{k \rightarrow+\infty} \frac{\ln p\left(\sigma_{\mid k}\right)}{\ln r\left(\sigma_{\mid k}\right)}=\alpha\right\}
\end{array}\right.
$$

then $E_{u}^{(\alpha)}=h_{u}^{-1}\left(K_{u}^{(\alpha)}\right)$. It is proved that we have for $f$ given by (16)

$$
\mu_{u}^{(\beta)}\left(K_{u}^{(\alpha)}\right)=\nu_{u}^{(\beta)}\left(E_{u}^{(\alpha)}\right)=1
$$

and

$$
\operatorname{HD}\left(K_{u}^{(\alpha)}\right)=\operatorname{HD}\left(E_{u}^{(\alpha)}\right)=f=\operatorname{HD}\left(\mu^{(\beta)}\right)=\operatorname{HD}\left(\nu^{(\beta)}\right) .
$$

Finally there are two cases for the multifractal analysis.

\section{Theorem 2.}

i) In the degenerate case: for all $(u, v) \in V^{2}$, for all $e \in E_{u v}$, $p(e)=\left(x_{u}^{-1} r(e) x_{v}\right)^{d}$. Then $\psi$ is linear and for all $\beta \in \mathbb{R}, \psi(\beta)=$ $d(1-\beta), \operatorname{HD}\left(K_{u}\right)=d=d_{\mu_{u}}$ and $K_{u}^{(\alpha)} \neq \varnothing$ if and only if $\alpha=d$.

ii) In the nondegenerate case: there exists $e \in E_{u v}, p(e) \neq$ $\left(x_{u}^{-1} r(e) x_{v}\right)^{d}$. Then $\psi$ is real analytic and strictly convex; $\alpha$ is a strictly decreasing function of $\beta$, i.e. $\alpha: \mathbb{R} \longrightarrow] \alpha_{\min }, \alpha_{\max }[; f$ is a strictly concave function of $\alpha$ and $K_{u}^{(\alpha)} \neq \varnothing$ if and only if $\alpha \in$ $\left[\alpha_{\min }, \alpha_{\max }\right]$.

\section{Thermodynamic formalism.}

This is a useful theory developped in [Bo], $[\mathrm{Ru}]$. It allows to transport some problems from the dynamical system $(\Lambda, \mu, T)$, where $T$ is for 
example a picewise $C^{1+\delta}$ expanding Markov map [R], onto a symbolic dynamical system $\left(\Sigma_{A}^{+}, \nu, \sigma\right)$ by a coding map.

\subsection{Symbolic dynamics.}

We introduce Markov partitions to make an analogy with the symbolic dynamical systems. In a sense, we replace small balls in the definition of dimension by small elements of the iterations of this partition by the expanding Markov map.

Let $\Lambda$ be a basic set, a $T$-invariant compact metric set. A Markov partition is a finite cover of $\Lambda: \mathcal{U}_{0}=\left(U_{1}, \ldots, U_{m}\right)$, consisting of proper rectangles (compact sets $R$ such that $R=\overline{\operatorname{int}(R)}$ ) which satisfy

- $\operatorname{int}\left(U_{i}\right) \cap \operatorname{int}\left(U_{j}\right)=\varnothing$ for $i \neq j$.

- Each $T\left(U_{i}\right)$ is a union of rectangles $U_{j}$.

We can construct Markov partitions of arbitrary small diameter. We then define the partition at the rank $n$ by

$$
\mathcal{U}_{n}=\bigvee_{i=0}^{n-1} T^{-i}\left(\mathcal{U}_{0}\right)
$$

We associate to this partition the transition matrix $A$ defined by

$$
A_{i, j}= \begin{cases}1, & \text { if } T^{-1}\left(\stackrel{\circ}{U_{j}}\right) \cap \stackrel{\circ}{U_{i}} \neq \varnothing, \quad 1 \leq i, j \leq p \\ 0, & \text { otherwise }\end{cases}
$$

which is irreducible (for all $(i, j)$, there exists $n$ such that $\left(A^{n}\right)_{i j}>0$ : you reach any $U_{i}$ from any $U_{j}$ ).

Consider the subshift of finite type associated to the matrix $A$

$$
\Sigma_{A}^{+}=\left\{\underline{x}=\left\{x_{n}\right\}_{n \geq 0} \in\{1, \ldots, m\}^{\mathbb{N}}: A_{x_{i} x_{i+1}}=1\right\},
$$

which is the set of admissible sequences.

We define the metric on $\Sigma_{A}^{+}($for $0<\lambda<1)$

$$
d^{\prime \prime}(\underline{x}, \underline{y})= \begin{cases}\lambda^{k}, & \text { if } k=\sup \left\{j: x_{i}=y_{i}, \text { for all } i, 0 \leq i<j\right\} \\ 0, & \text { if } \underline{x}=\underline{y}\end{cases}
$$


which is a compact set, and the shift $\sigma(\underline{x})=\underline{y}$, where for all $n \in \mathbb{N}$, $y_{n}=x_{n+1}$.

We then define a continuous (Lipschitz) surjection $\pi$,

$$
\begin{aligned}
\pi: \Sigma_{A}^{+} & \longrightarrow \Lambda \\
\underline{x} \longmapsto & \longmapsto \\
& \bigcap_{j \geq 0} T^{-j}\left(U_{x_{j}}\right)
\end{aligned}
$$

which is one-to-one on the set of points whose trajectories do not intersect the boundaries of the elements of the Markov partition (if not these points have no local dimension), a set of $\mu$ measure 1 when $\mu$ is a Gibbs measure. Nevertheless, it is bounded-to-one and satisfies $\pi \circ \sigma^{n}=T^{n} \circ \pi$.

\subsection{Thermodynamics.}

Let us define the following sets.

- Consider $M(\Lambda)$ (respectively $M\left(\Sigma_{A}^{+}\right)$) the set of Borel probability measures defined on $\Lambda$ (respectively $M\left(\Sigma_{A}^{+}\right)$).

- Let $M_{T}(\Lambda)$ (respectively $M_{\sigma}\left(\Sigma_{A}^{+}\right)$) be the set of $T$-invariant Borel probability measures on $\Lambda$ (respectively $\sigma$-invariant on $\Sigma_{A}^{+}$).

- Let $C(\Lambda)$ (respectively $C\left(\Sigma_{A}^{+}\right)$) be the set of continuous functions defined on $\Lambda$ (respectively $\Sigma_{A}^{+}$) and $C^{\delta}(\Lambda)$ (respectively $C^{\delta}\left(\Sigma_{A}^{+}\right)$) be the set of $\delta$-Hölder continuous functions.

The pressure of a function $\varphi \in C^{\delta}(\Lambda)$ (respectively $\bar{\varphi} \in C^{\delta}\left(\Sigma_{A}^{+}\right)$) is defined by

$$
P_{\varphi}=P_{T}(\varphi)=\sup _{\rho \in M_{T}(\Lambda)}\left(h_{\rho}+\int_{\Lambda} \varphi d \rho\right) \quad\left(=P_{\sigma}(\varphi \circ \pi)=P_{\sigma}(\bar{\varphi})\right)
$$

and the measures which achieve this supremum are called equilibrium measures. The entropy $h_{\rho}(T)$ - the Kolmogorov-Sinaï entropy of the map $T$ - is the following: define the set

$$
B(x, n, r)=\left\{y \in \Lambda: d\left(T^{i}(x), T^{i}(y)\right)<r, \text { for } 0 \leq i \leq n-1\right\},
$$

the set of points that cannot be distinguished from $x$ at the small distance $r$ after $(n-1)$ iterations. Then we get for an ergodic $T$-invariant probability measure $\mu$,

$$
h_{\mu}(T) \stackrel{\mu a . s .}{=}-\lim _{r \rightarrow 0} \varlimsup_{n \rightarrow+\infty} \frac{1}{n} \ln \mu(B(x, n, r)),
$$


which is a nonnegative real number in our case. Notice that the larger the entropy, the greater the rate of decrease of the indeterminacy of the dynamical system.

In our case, there exists a unique measure $\mu_{\varphi}$ (respectively $\nu_{\bar{\varphi}}, \mu_{\varphi}=$ $\left.\pi^{*} \nu_{\bar{\varphi}}\right)$ which is the Gibbs measure of the potential $\varphi$ (respectively $\bar{\varphi}$ ). The map $\pi:\left(\Sigma_{A}^{+}, \nu_{\bar{\varphi}}, s\right) \longrightarrow\left(\Lambda, \mu_{\varphi}, T\right)$ is an isomorphism of dynamical systems.

This means that the pullback of any Gibbs measure $\mu_{\varphi}$ on $\Lambda$ is a Gibbs measure $\nu_{\bar{\varphi}}$ on $\Sigma_{A}^{+}$. Conversely the pushforward of any Gibbs measure $\nu_{\bar{\varphi}}$ on $\Sigma_{A}^{+}$is a Gibbs measure $\mu_{\varphi}$ on $\Lambda$, and their thermodynamic quantities are equal: $P_{T}(\varphi)=P_{\sigma}(\varphi \circ \pi), h_{\mu_{\varphi}}(T)=h_{\nu_{\bar{\varphi}}}(\sigma)$.

The measure $\nu_{\bar{\varphi}}$ is well defined on the cylinders which generate the topology of $\Sigma_{A}^{+}$. There exist nonnegative constants $c$ and $C$ such that

$$
c \leq \frac{\nu_{\bar{\varphi}}\left\{\underline{y} \in \Sigma_{A}^{+}: y_{0}=x_{0}, \ldots, y_{n-1}=x_{n-1}\right\}}{\exp \left(-n P_{\bar{\varphi}}+\sum_{k=0}^{n-1} \bar{\varphi}\left(\sigma^{k}(\underline{x})\right)\right)} \leq C,
$$

uniformly in $n$.

The pressure function $P: C^{\delta}\left(\Sigma_{A}^{+}\right) \longrightarrow \mathbb{R}$ is real analytic (not true for arbitrary symbolics). Consider for $(\bar{\xi}, \bar{\zeta}) \in C^{\delta}\left(\Sigma_{A}^{+}\right)^{2}$, the map

$$
\begin{aligned}
Q: & \mathbb{R}^{2} \longrightarrow \mathbb{R} \\
& (x, y) \longmapsto P(x \bar{\xi}+y \bar{\zeta})
\end{aligned}
$$

It is real analytic in both variables, convex and strictly convex if and only if the functions $\bar{\xi}$ et $\bar{\zeta}$ are not conjugate to constants $c$ and $c^{\prime}$, i.e. $\bar{\xi} \neq c+\bar{\varphi}-\bar{\varphi} \circ \sigma, \bar{\varphi} \in C^{\delta}\left(\Sigma_{A}^{+}\right)$(respectively $\bar{\zeta}$ and $c^{\prime}$ ).

Let $\nu_{x_{0}} \bar{\xi}+y_{0} \bar{\zeta}$ be the Gibbs measure of the function $x_{0} \bar{\xi}+y_{0} \bar{\zeta} \in$ $C^{\delta}\left(\Sigma_{A}^{+}\right)$, then we have [M], [Ma], [MC], [R], [Ru], [Si1]

$$
\left\{\begin{array}{l}
\frac{\partial Q}{\partial x}\left(x_{0}, y_{0}\right)=\int_{\Sigma_{A}^{+}} \bar{\xi} d \nu_{x_{0} \bar{\xi}+y_{0} \bar{\zeta}} \\
\frac{\partial Q}{\partial y}\left(x_{0}, y_{0}\right)=\int_{\Sigma_{A}^{+}} \bar{\zeta} d \nu_{x_{0} \bar{\xi}+y_{0} \bar{\zeta}}
\end{array}\right.
$$




\section{Dimension spectrum and the thermodynamic theory.}

\subsection{Idea of the computation.}

Consider the Markov partition

$$
\mathcal{P}_{n}=\bigvee_{i=0}^{n-1} T^{-j}(\mathcal{P})
$$

where $\mathcal{P}=\left(K_{1}, K_{2}, \ldots, K_{q}\right)$ (see just below). The idea for computation of local dimensions $(1)$ is to replace small balls $B(x, r)$ by elements $V=T^{-n}(U) \in \mathcal{P}_{n}(U \in \mathcal{P})$ which are in the set $B_{j(\beta, n)}^{n}$ (see (29)) which cover at the limit the singularity set $C_{\alpha}$ for $\alpha=F^{\prime}(\beta)$. Those elements generate a measure $\mu_{\beta}$ (of course singular to each other) which is ergodic. We use the assumptions on $T$ and $\mu$ :

- For any $V=T^{-n}(U):=V(U) \in \mathcal{P}_{n}$ there exists an element $y(U) \in U$ such that

$$
\begin{aligned}
|V(U)| & =\left|T^{-n}(U)\right| \\
& =\left|\left(T^{-n}\right)^{\prime}(y(U))\right||U| \\
& =\exp \left(\sum_{j=0}^{n-1} J\left(T^{j}(y(U))\right)\right) \underbrace{|U|}_{\approx 1}
\end{aligned}
$$

(where the sign $\approx$ expresses that the ratios of both sides are uniformly bounded by constants), expression which controls the length of $V(U)$.

- Since the measure $\mu$ is a Gibbs measure we have following (20)

$$
\mu(V(U)) \approx \exp \left(\sum_{j=0}^{n-1} \xi\left(T^{j}(y(U))\right)\right.
$$

expression which controls the mass of $V(U)$.

It follows from the Birkhoff's sums and the ergodicity of the dy- 
namical system that

$$
\begin{aligned}
& \frac{\ln \mu(B(x, r))}{\ln r} \sim \frac{\ln \mu(V(U))}{\ln |V(U)|} \\
& \sim \frac{\frac{1}{n} \sum_{j=0}^{n-1} \xi\left(T^{j}(y(U))\right)}{\frac{1}{n} \sum_{j=0}^{n-1} J\left(T^{j}(y(U))\right)} \\
& \underset{n \rightarrow+\infty}{\mu_{\beta} a . s .} \frac{\int_{\Lambda} \xi d \mu_{\beta}}{\int_{\Lambda} J d \mu_{\beta}} \\
& =\alpha \\
& =F^{\prime}(\beta) \text {, }
\end{aligned}
$$

which gives the existence and the value of the local dimension for points covered by the sets of the type $B_{j(\beta, n)}^{n}(29)$. Otherwise it suffices to prove for the points which do not have this property that they do not have local dimension.

Note that it is not always possible to replace balls by elements of the partition [O2].

\subsection{Dimension spectrum.}

The Markov measures that are used are in fact a special case of Gibbs measures. These measures are associated to potentials $\bar{\varphi}$ depending only on the first coordinate, i.e. $\bar{\varphi}(\underline{x})=g\left(x_{0}\right)$ for $\underline{x}=\left(x_{i}\right)_{i \geq 0}$. For this purpose, consider the transfer operator

$$
\begin{aligned}
L_{\bar{\varphi}}: & C^{\delta}\left(\Sigma_{A}^{+}\right) \longrightarrow C^{\delta}\left(\Sigma_{A}^{+}\right) \\
& f \longmapsto \sum_{\underline{y} \in \sigma^{-1}(\underline{x})} \exp (\bar{\varphi}(\underline{y}) f(\underline{y})),
\end{aligned}
$$

and the corresponding operator defined on measures $L_{\bar{\varphi}}^{*}: M\left(\Sigma_{A}^{+}\right) \longrightarrow$ $M\left(\Sigma_{A}^{+}\right)$.

Then there exist (see $[\mathrm{Ru}])$ : 
i) $\lambda>0(=\exp (P(\bar{\varphi})))$,

ii) $h \in C^{0}\left(\Sigma_{A}^{+}\right)$such that $h>0$,

iii) $\rho \in M\left(\Sigma_{A}^{+}\right)$,

such that $L_{\bar{\varphi}}(h)=\lambda h, L_{\bar{\varphi}}^{*}(\rho)=\lambda \rho$ and $\nu_{\bar{\varphi}}=h \rho \in M_{\sigma}\left(\Sigma_{A}^{+}\right)$ $\left(d \nu_{\bar{\varphi}}=h\left(x_{0}\right) d \rho\right)$ which is the Gibbs measure for $\bar{\varphi}$ and can be represented on the cylinder sets by

$$
\begin{aligned}
\nu_{\bar{\varphi}}\left\{\underline{y} \in \Sigma_{A}^{+}: y_{0}=x_{0}, \ldots, y_{n}=x_{n}\right\} \\
=R\left(x_{0}, x_{1}\right) R\left(x_{1}, x_{2}\right) \cdots R\left(x_{n-1}, x_{n}\right) p\left(x_{n}\right),
\end{aligned}
$$

where we have

$$
R\left(x_{i}, x_{j}\right)=\frac{A_{i j} h\left(x_{i}\right) \exp \left(\bar{\varphi}\left(x_{i}\right)\right)}{\lambda h\left(x_{j}\right)}
$$

and $p$ is an invariant probability vector: $\sum_{i} p_{i}=1$ and $R(p)=p$.

These equations define all the Markov measures $\nu_{u}$ and a fortiori all the measures $\mu_{u}$.

We compute the partition functions (7) for any pair $(k, s) \in \mathbb{N}^{*} \times \mathbb{R}$,

$$
Z_{k}(s)=\sum_{V(U) \in \mathcal{P}_{k}} \mu(V(U))^{s}=\sum_{\substack{u \in E \\ p(u)>0}} \sum_{\gamma \in E_{u}^{(k)}} p(\gamma)^{s} .
$$

Let $C(s)=\max _{u \in E} \mathbb{P}\left(X_{0}=u\right)^{s}$ and for any pair $(k, m)$ of integers, we have

$$
Z_{k}(s)=\sum_{u \in E} \sum_{\gamma \in E_{u}^{(k)}} p(\gamma)^{s} \mathbb{P}\left(X_{0}=u\right)^{s}
$$

and

$$
Z_{m}(s)=\sum_{v \in E} \sum_{\gamma^{\prime} \in E_{v}^{(m)}} p\left(\gamma^{\prime}\right)^{s} \mathbb{P}\left(X_{0}=v\right)^{s} .
$$

We then obtain

$$
\begin{aligned}
Z_{k}(s) & Z_{m}(s) \\
= & \sum_{u \in E} \sum_{v \in E} \sum_{\left(\gamma, \gamma^{\prime}\right) \in E_{u}^{(k)} \times E_{v}^{(m)}}\left[p(\gamma) p\left(\gamma^{\prime}\right)\right]^{s}\left[\mathbb{P}\left(X_{0}=u\right) \mathbb{P}\left(X_{0}=v\right)\right]^{s} \\
\leq & C(s) Z_{k+m}(s) \\
= & C(s) \sum_{u \in E} \sum_{v \in E} \sum_{\gamma^{\prime \prime} \in E_{u}^{(k+m)}} p\left(\gamma^{\prime \prime}\right)^{s} \mathbb{P}\left(X_{0}=u\right)^{s},
\end{aligned}
$$


where $\gamma^{\prime \prime}=\gamma \gamma^{\prime}: \gamma=u e_{2} \cdots e_{k}$ and $\gamma^{\prime}=v e_{2}^{\prime} \cdots e_{m}^{\prime}$. Finally we obtain

$$
\frac{1}{C(s)} Z_{k}(s) \frac{1}{C(s)} Z_{m}(s) \leq \frac{1}{C(s)} Z_{k+m}(s),
$$

which implies that the sequence $\left\{\ln \left(Z_{k}(s) / C(s)\right\}_{k \geq 1}\right.$ is subadditive, and that the sequence $\left\{\ln Z_{k}(s) / k\right\}_{k>1}$ converges to a concave function.

Following the same method we prove (8) that for any pair $(s, t)$ of real numbers the sequence

$$
-\frac{1}{k} \ln G_{\mathcal{D}}^{(k)}(s, t) \underset{k \rightarrow+\infty}{\longrightarrow} G(s, t),
$$

where

$$
G_{\mathcal{D}}^{(k)}(s, t)=\sum_{u \in E} \sum_{\gamma \in E_{u}^{(k)}} p(\gamma)^{s} \mathbb{P}\left(X_{0}=u\right)^{s}|J(\gamma)|^{t}
$$

(we haved assumed that $\left|J_{u}\right|=1$ for any $u \in E$ ).

Framework. The dynamical systems $\left(K_{u}, \mu_{u}, H\right)_{u \in E}$ (respectively $\left.\left(E_{u}, \nu_{u}, \sigma\right)_{u \in E}\right)$ may be studied in the same way. Define $(\mathbf{K}, \mu, T)$ (respectively $(\mathbf{E}, \nu, \sigma))$ be one of these sets, where the map $T$ is a picewise $C^{\infty}$ expanding Markov map $\left(T=H^{-1}\right.$, for all $\left.e \in E, T_{e}^{-1}=H_{e}\right)$.

The measure $\mu$ is the Gibbs measure of the potential $\xi \in C^{\delta}(\mathbf{K})<0$ (respectively $\bar{\xi} \in C^{\delta}(\mathbf{E})$ ), and $J=-\ln T \in C^{\delta}(\mathbf{K})<0$ (respectively $\left.\bar{J} \in C^{\delta}(\mathbf{E})\right)$. We have seen that for Markov measures the associated potentials $\bar{J}$ and $\bar{\xi}$ depends only on the first coordinate.

We now prove theorem $\mathrm{A}$.

Assume that $P(\xi)=0$, if not take $\widetilde{\xi}=\xi-P(\xi)$ which is cohomologous to the potential $\xi$, which implies the equality $\mu_{\widetilde{\xi}}=\mu_{\xi}=\mu$.

From the expressions (23) and (24) there exists for any set $V(U)=$ $T^{-n}(U) \in \mathcal{P}_{n}$ an element $y(U) \in U \subset \mathcal{P}$ such that

$$
\begin{aligned}
& \frac{1}{n} \ln \mu(V(U)) \sim \frac{1}{n} \sum_{j=0}^{n-1} \xi\left(T^{j}(y(U))\right), \\
& \frac{1}{n} \ln |V(U)| \sim \frac{1}{n} \sum_{j=0}^{n-1} J\left(T^{j}(y(U))\right) .
\end{aligned}
$$

Since the functions $J$ and $\xi$ are $C^{\delta}$-Hölder, they are continuous on the compact set $K$ and therefore take their values in compact sets $[a, b]$ and $[c, d]$. 
Consider for any integer $i \in \mathbb{Z} \cap[a n, b n-1]$ (linear scale) the set

$$
A_{i}^{n}=\left\{V(U) \in \mathcal{P}_{n}: \ln \mu(V(U)) \in[i, i+1[\},\right.
$$

and for any real number $\beta$, the integer $i(\beta, n)$ such that

$$
\sum_{V(U) \in A_{i}^{n}} \mu(V(U))^{\beta} \leq \sum_{V(U) \in A_{i(\beta, n)}^{n}} \mu(V(U))^{\beta} .
$$

Since there is a linear scale we have for any real number $\beta$,

$$
\begin{aligned}
\sum_{V(U) \in A_{i(\beta, n)}^{n}} \mu(V(U))^{\beta} & \leq \sum_{i} \sum_{V(U) \in A_{i}^{n}} \mu(V(U))^{\beta} \\
& =\sum_{V(U) \in \mathcal{P}_{n}} \mu(V(U))^{\beta} \\
& =Z_{n}(\beta) \\
& \leq(b-a) n \sum_{V(U) \in A_{i(\beta, n)}^{n}} \mu(V(U))^{\beta} .
\end{aligned}
$$

We get therefore for any real number $\beta(7)$,

$$
\begin{aligned}
\frac{1}{n} \ln Z_{n}(\beta) & \sim \frac{1}{n} \ln \left(\sum_{V(U) \in A_{i(\beta, n)}^{n}} \mu(V(U))^{\beta}\right) \\
& \sim \beta \frac{i(\beta, n)}{n}+\frac{\ln \# A_{i(\beta, n)}^{n}}{n},
\end{aligned}
$$

since the elements of $A_{i(\beta, n)}^{n}$ have same mass $\approx \exp (i(\beta, n))$.

Among the elements of $A_{i(\beta, n)}^{n}$ we make a new selection for the length, in order to obtain elements of $A_{i(\beta, n)}^{n}$ with same mass and same length.

Therefore consider in the same way for all integer $j \in \mathbb{Z} \cap[c n, d n-$ 1] (linear scale) the set

$$
B_{j}^{n}=\left\{V(U) \in A_{i(\beta, n)}^{n}: \ln |V(U)| \in[j, j+1[\} .\right.
$$

For any real number $\beta$, define the integer $j(\beta, n)$ such that

$$
\sum_{V(U) \in B_{j}^{n}} \mu(V(U))^{\beta} \leq \sum_{V(U) \in B_{j(\beta, n)}^{n}} \mu(V(U))^{\beta} .
$$


We then have for any real number $\beta$,

$$
\begin{aligned}
\sum_{V(U) \in B_{j(\beta, n)}^{n}} \mu(V(U))^{\beta} & \leq \sum_{j} \sum_{V(U) \in B_{j}^{n}} \mu(V(U))^{\beta} \\
& =\sum_{V(U) \in A_{i(\beta, n)}^{n}} \mu(V(U))^{\beta} \\
& \leq(d-c) n \sum_{V(U) \in B_{j(\beta, n)}^{n}} \mu(V(U))^{\beta},
\end{aligned}
$$

which implies for any real number $\beta$,

$$
\frac{1}{n} \ln \left(\sum_{V(U) \in A_{i(\beta, n)}^{n}} \mu(V(U))^{\beta}\right) \sim \frac{1}{n} \ln \left(\sum_{V(U) \in B_{j(\beta, n)}^{n}} \mu(V(U))^{\beta}\right) .
$$

Finally we have

$$
\begin{aligned}
-\frac{1}{n} \ln _{b(n(\beta))} Z_{n}(\beta) & \sim-\frac{1}{n} \ln _{b(n(\beta))}\left(\sum_{V(U) \in B_{j(\beta, n)}^{n}} \mu(V(U))^{\beta}\right) \\
& \sim \beta \frac{i(\beta, n)}{j(\beta, n)}+\frac{\ln \# B_{j(\beta, n)}^{n}}{j(\beta, n)} .
\end{aligned}
$$

Notice that the set $B_{j(\beta, n)}^{n} \subset A_{i(\beta, n)}^{n}$ consists of elements of the partition $\mathcal{P}_{n}$ with "same" measure $\exp (i(\beta, n))$ and "same" length $\exp (j(\beta, n))$ $=b(n(\beta))^{-n}$ (in the order $\left.(1 / n) \ln \right)$, where $b(n(\beta))$ is the logarithmic basis in the expression of the free energy function (6),

$$
\left\{\begin{array}{l}
\mu(V(U)) \approx \exp (i(\beta, n)), \\
|V(U)| \approx \exp (j(\beta, n)),
\end{array} \quad \text { for all } V(U) \in B_{j(\beta, n)}^{n} .\right.
$$

In fact it is the set where the distribution of the mass $\mu(V(U))^{\beta}$ of the function is the largest, and this is where large deviations occur.

The aim is to determine the measures $\mu_{\beta}$ whose supports are the singularity sets $C_{\alpha}$. We consider for any real number $\beta$ the following probability measures

$$
\theta_{n}(\beta)=\frac{1}{\# B_{j(\beta, n)}^{n}} \sum_{V(U) \in B_{j(\beta, n)}^{n}} \delta_{y(U)} \quad \text { and } \quad \zeta_{n}(\beta)=\frac{1}{n} \sum_{j=0}^{n-1} T^{j} \theta_{n}(\beta)
$$


(We remark that a cluster point of the sequence $\left\{\zeta_{n}(\beta)\right\}_{n \geq 1}$ is $T$-invariant.)

By our assumptions, the following sequences take their values in compact sets

$$
\begin{gathered}
\frac{1}{n} \ln \# B_{j(\beta, n)}^{n} \in[-d,-c], \quad \frac{i(\beta, n)}{n} \in[a, b], \\
\frac{j(\beta, n)}{n} \in[c, d], \quad \zeta_{n}(\beta) \in M(\mathbf{K}) .
\end{gathered}
$$

Then there exists a sub-sequence $\left\{n_{k}\right\}_{k \geq 1}$, that we note for simplicity $\{m\}_{m \geq 1}(m=m(\beta))$, such that

$$
\left\{\begin{array}{l}
\frac{1}{m} \ln \# B_{j(\beta, m)}^{m} \underset{m \rightarrow+\infty}{\longrightarrow} \gamma(\beta) \in[-d,-c]>0, \\
\frac{i(\beta, m)}{m} \underset{m \rightarrow+\infty}{\longrightarrow} \eta(\beta) \in[a, b]<0, \\
\frac{j(\beta, m)}{m} \underset{m \rightarrow+\infty}{\longrightarrow}-b(\beta) \in[c, d]<0, \\
\zeta_{m}(\beta) \in M(\mathbf{K})_{m \rightarrow+\infty}^{\longrightarrow} \zeta_{\beta} \in M_{T}(\mathbf{K}) .
\end{array}\right.
$$

We get finally with (30) for any real number $\beta$,

$$
-\frac{1}{m} \ln _{b(m(\beta))} Z_{m}(\beta)=F_{m}(\beta) \underset{m \rightarrow+\infty}{\longrightarrow} \frac{-1}{b(\beta)}(\gamma(\beta)+\beta \eta(\beta)),
$$

where $\gamma(\beta)$ and $-\eta(\beta)$ represent entropies and $b(\beta)$ a Lyapunov exponent.

Consider the functional

$$
\begin{aligned}
I: M_{T}(\mathbf{K}) \times \mathbb{R} \longrightarrow \mathbb{R} \\
(\rho, \beta) \longmapsto \frac{h_{\rho}(T)+\beta \int_{\mathbf{K}} \xi d \rho}{\int_{\mathbf{K}} J d \rho} .
\end{aligned}
$$

We have the following fundamental result. 
Lemma 1 ([Si1]). We have for any real number $\beta$,

$$
F(\beta)=\inf _{\rho \in M_{T}(\mathbf{K})}(I(\rho, \beta))=\inf _{\substack{\rho \in M_{T}(\mathbf{K}) \\ \rho \operatorname{ergodic}}}(I(\rho, \beta)) .
$$

The proof is given in three steps (the three following expressions):

1) For all $\beta \in \mathbb{R}, \sup _{\rho \in M_{T}(\mathbf{K})}(-I(\rho, \beta))=\sup _{\rho \in M_{T}(\mathbf{K})}(-I(\rho, \beta))$. $\rho$ ergodic

2) For all $\beta \in \mathbb{R}, \varliminf_{n \rightarrow+\infty}-F_{n}(\beta) \geq \sup _{\substack{\rho \in M_{T}(\mathbf{K}) \\ \rho \text { ergodic }}}(-I(\rho, \beta))$.

3) For all $\beta \in \mathbb{R}, \varlimsup_{n \rightarrow+\infty}-F_{n}(\beta) \leq \sup _{\rho \in M_{T}(\mathbf{K})}(-I(\rho, \beta))$.

The functional $I$ is semicontinuous since the (entropy) map $\rho \longmapsto$ $h_{\rho}(T)$ is expanding, i.e. two orbits never stay $\varepsilon$-close. Its infimum is attained since $M_{T}(\mathbf{K})$ is a compact set. Since the ergodic measures are extremal and form a $G_{\delta}$ set in the convex set $M_{T}(\mathbf{K})$, we have the first equality. The two others are much harder to prove.

For the second step we consider an ergodic Borel probability measure $\rho \in M_{T}(\mathbf{K})$. The ergodic theorem implies that for $\rho$ allmost every $x$,

$$
\frac{1}{n} \sum_{j=0}^{n} \delta_{T^{j}(x)} \underset{n \rightarrow+\infty}{\longrightarrow} \rho .
$$

We know that for $\bar{\rho}$ (where $\bar{\rho} \leftrightarrow \rho$ ) allmost cylinders the ergodic measure $\bar{\rho}$ satisfies: $\bar{\rho}\left(C_{n}(\underline{x})\right) \approx e^{-n h_{\bar{\rho}}(\sigma)}$ and $\left|C_{n}(\underline{x})\right| \approx e^{-n \chi_{\bar{\rho}}(\sigma)}$. For the elements of the Markov partition (which correspond on the dynamical system to the cylinders) $V(U) \in \mathcal{P}_{n}$, we have

$$
\rho(V(U)) \approx e^{-n h_{\rho}(T)} \quad \text { and } \quad|V(U)| \approx e^{-n \chi_{\rho}(T)} .
$$

Using the sets $B_{j(\beta, n)}(29)$ we see that (31)

$$
\left\{\begin{array}{l}
\frac{i(\beta, n)}{n} \underset{n \rightarrow+\infty}{\longrightarrow} \int_{\mathbf{K}} \xi d \rho=-h_{\rho}(T), \\
\frac{j(\beta, n)}{n} \underset{n \rightarrow+\infty}{\longrightarrow} \int_{\mathbf{K}} J d \rho=-\chi_{\rho}(T) .
\end{array}\right.
$$


According the Shannon-McMillan-Breiman theorem [DGS, p. 81] we define for $\varepsilon>0$ the set

$$
H_{(\beta, \rho, n, \varepsilon)}=\left\{V(U) \in \mathcal{P}_{n}:-n \chi_{\rho}(T)-\varepsilon<j(\beta, n)<-n \chi_{\rho}(T)+\varepsilon\right\},
$$

for which there exists an integer $N$ such that for any integer $n \geq N$, we get

$\rho\left(H_{(\beta, \rho, n, \varepsilon)}\right) \geq 1-\varepsilon \quad$ and $\quad \# H_{(\beta, \rho, n, \varepsilon)} \geq(1-\varepsilon) \exp \left(n\left(h_{\rho}(T)-\varepsilon\right)\right)$.

We get therefore for any real number $\beta$ and any element $V(U) \in$ $H_{(\beta, \rho, n, \varepsilon)}$,

$$
\rho(V(U))^{\beta} \geq \exp \left(\beta n\left(\int_{\mathbf{K}} \xi d \rho-\varepsilon\right)\right)
$$

( $\pm \varepsilon$ according to the sign of the real number $\beta$ ), which gives for any integer $n \geq 1$,

$$
\begin{aligned}
-F_{n}(\beta) & =\frac{1}{n} \ln _{b(n(\beta))} Z_{n}(\beta) \\
\geq & \frac{1}{n} \ln _{b(n(\beta))}\left(\sum_{V(U) \in H_{(\beta, \rho, n, \varepsilon)}} \rho(V(U))^{\beta}\right) \\
& \geq \frac{\ln \# H_{(\beta, \rho, n, \varepsilon)}+\beta \frac{\int_{\mathbf{K}} \xi d \rho+\varepsilon}{\int_{\mathbf{K}}-J d \rho+\varepsilon}-J d \rho+\varepsilon}{\int_{\mathbf{K}}} \\
& \geq \frac{h_{\rho}(T)+\beta \int_{\mathbf{K}} \xi d \rho-2 \varepsilon}{\int_{\mathbf{K}}-J d \rho+\varepsilon}
\end{aligned}
$$

which implies that

$$
\varliminf_{n \rightarrow+\infty}-F_{n}(\beta) \geq \frac{h_{\rho}(T)+\beta \int_{\mathbf{K}} \xi d \rho}{\int_{\mathbf{K}}-J d \rho}=-I(\rho, \beta),
$$

which ends the second step since the ergodic measure $\rho$ is arbitrary. 
For the third step, using (23), (24) and (26), we compute for any real number $\beta$ the following integrals

$$
\left\{\begin{array}{l}
\int_{\mathbf{K}} J d \zeta_{m}(\beta)=\frac{1}{\# B_{j(\beta, m)}^{m}} \sum_{V(U) \in B_{j(\beta, m)}^{m}}(\frac{1}{m} \underbrace{\sum_{j=0}^{m-1} J\left(T^{j}(y(U))\right)}_{=\ln |V(U)|(23)}), \\
\int_{\mathbf{K}} \xi d \zeta_{m}(\beta)=\frac{1}{\# B_{j(\beta, m)}^{m}} \sum_{V(U) \in B_{j(\beta, m)}^{m}}(\frac{1}{m} \underbrace{\sum_{j=0}^{m-1} \xi\left(T^{j}(y(U))\right)}_{=\ln \mu(V(U))(24)} .
\end{array}\right.
$$

Using (32) and (33) we have

$$
\left\{\begin{array}{l}
\frac{i(\beta, m)}{m} \underset{m \rightarrow+\infty}{\longrightarrow} \eta(\beta)=\int_{\mathbf{K}} \xi d \zeta_{\beta}, \\
\frac{j(\beta, m)}{m}=-b(\beta, m) \underset{m \rightarrow+\infty}{\longrightarrow}-b(\beta)=\int_{\mathbf{K}} J d \zeta_{\beta} .
\end{array}\right.
$$

We get finally for any real number $\beta$,

$$
\frac{1}{m} \ln _{b(m(\beta))} Z_{m}(\beta)=-F_{m}(\beta) \underset{m \rightarrow+\infty}{\rightarrow} \frac{\gamma(\beta)+\beta \int_{\mathbf{K}} \xi d \zeta_{\beta}}{\int_{\mathbf{K}}-J d \zeta_{\beta}}
$$

In this expression we do not know the value $\gamma(\beta)$ which satisfies the following.

Lemma 2. For all $\beta \in \mathbb{R}, \gamma(\beta) \leq h_{\zeta_{\beta}}$.

This estimate uses a standard argument of Misiurewicz [DGS, p. 145].

It implies that (34) becomes for any real number $\beta$,

$$
-F_{m}(\beta) \leq\left(-I\left(\zeta_{\beta}, \beta\right)\right),
$$

which implies that

$$
-F_{m}(\beta) \leq \sup _{\rho \in M_{T}(\mathbf{K})}(-I(\rho, \beta))
$$


Remember that the sequence $\left\{-F_{m}(\beta)\right\}_{m \geq 1}$ is a subsequence (32), which implies that

$$
\varlimsup_{n \rightarrow+\infty}-F_{n}(\beta) \leq \sup _{\rho \in M_{T}(\mathbf{K})}(-I(\rho, \beta))
$$

which ends the third step and the proof of Lemma 1.

By the same way we prove that for any pair $(x, y) \in \mathbb{R}^{2}$ we have (35)

$$
G_{\mathcal{D}}(x, y)=P(x \xi+y J)=\sup _{\rho \in M_{T}(\mathbf{K})}\left(h_{\rho}(T)+\int_{\mathbf{K}}(x \xi+y J) d \rho\right) .
$$

This function is real analytic in both variables, and by the way it is computed we have

$$
G_{D}(s, t)=\ln \phi(s, t) .
$$

Finally define the Gibbs measure $\mu_{\beta}$ associated to the potential $\xi_{\beta}=$ $\beta \xi-F(\beta) J$. We verify that we have for any real number $\beta$,

$$
P\left(\xi_{\beta}\right)=P(\beta \xi-F(\beta) J)=\sup _{\rho \in M_{T}(\mathbf{K})}\left(h_{\rho}(T)+\int_{\mathbf{K}} \xi_{\beta} d \rho\right)=0 .
$$

It implies that the unique measure which achieves the value 0 is the Gibbs measure $\mu_{\beta}$. Replacing this result in the expression of the free energy function, we obtain

$$
F(\beta)=\inf _{\rho \in M_{T}(\mathbf{K})}\left(\frac{h_{\rho}(T)+\beta \int_{\mathbf{K}} \xi d \rho}{\int_{\mathbf{K}} J d \rho}\right)=\frac{h_{\mu_{\beta}}(T)+\beta \int_{\mathbf{K}} \xi d \mu_{\beta}}{\int_{\mathbf{K}} J d \mu_{\beta}},
$$

for all $\beta \in \mathbb{R}$. Since we have for any real number $\beta$,

$$
G_{\mathcal{D}}(\beta, \psi(\beta))=\ln \phi(\beta, \psi(\beta))=0=G_{\mathcal{D}}(\beta,-F(\beta)),
$$

we have $F=-\psi$, which ends the proof of Theorem A.

Since the pressure is differentiable (36), by differentiating the following expression

$$
P(\beta \xi-F(\beta) J)=0,
$$


we get for any real number $\beta$ (22),

$$
\frac{\partial P}{\partial x}(\beta,-F(\beta))=\int_{\mathbf{K}} \xi d \mu_{\beta}<0
$$

and

$$
\frac{\partial P}{\partial y}(\beta,-F(\beta))=\int_{\mathbf{K}} J d \mu_{\beta}<0 .
$$

We then obtain for any real number $\beta$,

$$
F^{\prime}(\beta)=\frac{\int_{\mathbf{K}} \xi d \mu_{\beta}}{\int_{\mathbf{K}} J d \mu_{\beta}}>0 .
$$

Differentiating once more, we obtain for any real number $\beta[\mathrm{M}]$, [Ma], $[\mathrm{R}],[\mathrm{Si} 1]$,

$$
F^{\prime \prime}(\beta)=\frac{F^{\prime}(\beta)^{2}\left(\frac{\partial^{2} P}{\partial y^{2}}\right)-2 F^{\prime}(\beta)\left(\frac{\partial^{2} P}{\partial x \partial y}\right)+\left(\frac{\partial^{2} P}{\partial x^{2}}\right)}{\left(\frac{\partial P}{\partial x}\right)}(\beta,-F(\beta)) \leq 0 .
$$

We prove that $F^{\prime \prime}<0$ if and only if the functions $\xi$ et $J$ are not cohomologous to constants [Ru] (if not $F$ is linear).

Consider the Legendre-Fenchel transform of $F(5)$. Since $F$ is at least $C^{1}$ (it is real analytic) and according to the theory of conjugate functions $[\mathrm{E}]$, we have for the function $f$ and any real number $\beta$,

$$
f(\alpha)+F(\beta)=\alpha \beta \quad \text { if and only if }\left\{\begin{array}{c}
\alpha=F^{\prime}(\beta), \\
\beta=f^{\prime}(\alpha) .
\end{array}\right.
$$

We then obtain (37) for any real number $\beta$,

$$
f\left(F^{\prime}(\beta)\right)=\beta F^{\prime}(\beta)-F(\beta)=\frac{h_{\mu_{\beta}}(T)}{\chi_{\mu_{\beta}}(T)}=\frac{\int_{\mathbf{K}} \xi_{\beta} d \mu_{\beta}}{\int_{\mathbf{K}} J d \mu_{\beta}}=d_{\mu_{\beta}} .
$$

In the degenerate case, the free energy function $F$ is linear $\beta \longmapsto d_{\mu}(\beta-$ 1 ), and the dimension spectrum $f \equiv d=d_{\mu}=\operatorname{HD}(\mu)$. 
If not the free energy function is strictly increasing and strictly concave. This implies in particular that the dimension spectrum $f$ is real analytic on the interval $] \alpha_{\min }, \alpha_{\max }[$ where

$$
\left\{\begin{array}{l}
\alpha_{\min }=\inf _{\beta \in \mathbb{R}} F^{\prime}(\beta)=\lim _{\beta \rightarrow+\infty} F^{\prime}(\beta), \\
\alpha_{\max }=\sup _{\beta \in \mathbb{R}} F^{\prime}(\beta)=\lim _{\beta \rightarrow-\infty} F^{\prime}(\beta),
\end{array}\right.
$$

and strictly concave since for any $\left.\alpha=F^{\prime}(\beta) \in\right] \alpha_{\min }, \alpha_{\max }[$,

$$
f^{\prime \prime}(\alpha)=\frac{1}{F^{\prime \prime}(\beta)}<0 \text {. }
$$

In the expression (32) and the existence of the limit $F(\beta)$, we have for any real number $\beta, \zeta_{\beta}=\mu_{\beta}$. The sets $B_{j(\beta, n)}^{n}$ from (29) cover at the limit the singularity set $C_{\alpha}$ where $\alpha=\alpha(\beta)=F^{\prime}(\beta)$ (see Section 4.1).

We can prove directly [CLP], [Si1] that $f(\alpha)=\operatorname{HD}\left(C_{\alpha}\right)$. Here we have parametrized all the fractal sets $\left\{C_{\alpha(\beta)}\right\}_{\beta \in \mathbb{R}}$, and we have associated to the Gibbs measure $\mu$ a family of Gibbs measures $\left\{\mu_{\beta}\right\}_{\beta \in \mathbb{R}}$ (respectively $\nu$ and the family $\left\{\nu_{\beta}\right\}_{\beta \in \mathbb{R}}$ ) where $\mu_{\beta}$ has the potential $\beta \xi-F(\beta) J \in C^{\delta}(\mathbf{K})$ (respectively $\beta \bar{\xi}-F(\beta) \bar{J} \in C^{\delta}(\mathbf{E})$ ).

Let $\mu_{-\infty}$ (respectively $\mu_{+\infty}$ ) be a cluster point of the $\mu_{\beta}$ when $\beta \longrightarrow-\infty$ (respectively $\beta \longrightarrow+\infty$ ) - respectively $\nu_{-\infty}$ and $\nu_{+\infty}$ in $M_{\sigma}(\mathbf{E})$. It is clear with (13) that we obtain the extremal points $\alpha_{ \pm \infty}$ given in (39) and the corresponding singularity sets $C_{\alpha_{ \pm \infty}}$. Remark that the way there are given they may be not well defined. But in Section 6 we see that they are uniquely determined. tion 2).

We have thus proved Theorem B which contains Theorem 2 (Sec-

REMARKS. 1) We have:

- $F(0)=-\operatorname{HD}(\mathbf{K})=d ; f\left(F^{\prime}(0)\right)=\sup f(\alpha)=d$.

- $F(1)=0 ; f\left(F^{\prime}(1)\right)=F^{\prime}(1)$ and the tangeant of the graph $\alpha \longmapsto$ $f(\alpha)$ at the point $\alpha=F^{\prime}(1)=d_{\mu}$ is the line $y=x$. Moreover we have $\mu_{1}=\mu$.

2) For any $\beta \in \overline{\mathbb{R}}$ and $\alpha=F^{\prime}(\beta)$ we have $\mu_{\beta}\left(C_{\alpha}\right)=1$ (therefore the $\mu_{\beta}$ are singular to each other), the measure $\mu_{\beta}$ is exact dimensional since $d_{\mu_{\beta}}=\operatorname{HD}\left(\mu_{\beta}\right)=f(\alpha)$. The tangent of the graph $\alpha \longmapsto f(\alpha)$ at the point $\alpha=\alpha(\beta)=F^{\prime}(\beta)$ is the line $y=\beta x-F(\beta)$ (41). The measure $\mu$ is also exact dimensional since $\mu_{1}=\mu$. 


\section{Multifractal spectra of entropy and Lyapunov.}

The multifractal spectra of entropy, (9) and (10), and Lyapunov exponents, (11) and (12), are given by the following. $\left.\Sigma_{A}^{+}\right)$

Let us define (there are same values when using the subshift $\mathbf{E}=$

$$
\begin{aligned}
& \eta_{\min }=\inf _{\beta \in \mathbb{R}} \int_{\mathbf{K}}-\xi d \mu_{\beta}=\eta_{+\infty}, \\
& \eta_{\max }=\sup _{\beta \in \mathbb{R}} \int_{\mathbf{K}}-\xi d \mu_{\beta}=\eta_{-\infty}, \\
& \vartheta_{\min }=\inf _{\beta \in \overline{\mathbb{R}}} \int_{\mathbf{K}}-J d \mu_{\beta}=\vartheta_{+\infty}, \\
& \vartheta_{\max }=\sup _{\beta \in \mathbb{R}} \int_{\mathbf{K}}-J d \mu_{\beta}=\vartheta_{-\infty} .
\end{aligned}
$$

In the degenerate case for the dimension spectrum, the two spectra are simultaneously degenerate: hence the functions $\xi$ and $J$ are cohomologous to constants. In this situation the two intervals $\left[\eta_{\min }, \eta_{\max }\right]$ and $\left[\vartheta_{\min }, \vartheta_{\max }\right]$ are reduced to points $h_{\mu}$ and $\chi_{\mu}$.

Otherwise at least one of the two spectra is not degenerate. This means that at least two of the three spectra (plus dimension spectrum) are not degenerate, and therefore one of the functions $E_{n}(10)$ and $L_{y}$ (12) is real analytic on an open interval.

Proof of Theorem C. Suppose that for some $\eta \notin\left[\eta_{\min }, \eta_{\max }\right]$ we have $\mathbf{E}(\eta) \neq \varnothing(10)$. The concentration of the measures $\nu_{\bar{\xi}}$ and $\nu_{\bar{J}}$ are given on $\mathbf{E}$ by expansions of the type (26)

$$
\sum_{j=0}^{n-1} \bar{\xi}\left(\sigma^{j}(\underline{x})\right) \quad \text { and } \quad \sum_{j=0}^{n-1} \bar{J}\left(\sigma^{j}(\underline{x})\right) .
$$

For any $\underline{x} \in \mathbf{E}(\eta)$ we have

$$
-\frac{1}{n} \sum_{j=0}^{n-1} \bar{\xi}\left(\sigma^{j}(\underline{x})\right) \underset{n \rightarrow+\infty}{\longrightarrow} \eta
$$


and for any $\beta \in \overline{\mathbb{R}}, \nu_{\beta}(\mathbf{E}(\eta))=0$ since $\eta \notin\left[\eta_{\min }, \eta_{\max }\right]$ because the last expression converges to

$$
\int_{\mathbf{E}}-\bar{\xi} d \nu_{\beta} \in\left[\eta_{\min }, \eta_{\max }\right]
$$

We obtain in the same way the following convergence

$$
-\frac{1}{n} \sum_{j=0}^{n-1} \bar{J}\left(\sigma^{j}(\underline{x})\right) \underset{n \rightarrow+\infty}{\longrightarrow} \vartheta \notin\left[\vartheta_{\min }, \vartheta_{\max }\right] .
$$

We have on a set $\Omega$ the existence of local dimension : for all $x \in \Omega$, $d_{\nu}(\underline{x})=\eta / \vartheta$. On the other hand we have for any $\beta \in \overline{\mathbb{R}}, \nu_{\beta}(\Omega)=0$ implies $\mathbf{E}(\eta) \subset\left\{x: d_{\nu}(\underline{x})\right.$ does not exist $\}$, which gives a contradiction.

In fact the sequences in (45) are in the domain of attraction of the measure $\nu_{\beta}$, and therefore we have

$$
(\eta, \vartheta)=\left(\int_{\mathbf{E}}-\bar{\xi} d \nu_{\beta}, \int_{\mathbf{E}}-\bar{J} d \nu_{\beta}\right) \quad\left(=\left(\int_{\mathbf{K}}-\xi d \mu_{\beta}, \int_{\mathbf{K}}-J d \mu_{\beta}\right)\right) .
$$

Then we obtain for $\alpha=F^{\prime}(\beta)$ the spectra (10) and (12)

$$
E(\eta)=L(\vartheta)=C_{\alpha}
$$

and

$$
E_{n}(\eta)=L_{y}(\vartheta)=\operatorname{HD}\left(C_{\alpha}\right)=f(\alpha)=d_{\mu_{\beta}}
$$

which gives Theorem C.

\section{Multi-multifractal, extremal measures and graphs.}

In the multifractal analysis of a measure $\mu$ the support $\mathbf{K}$ is decomposed into fractal sets which represent the singularity sets (level sets for local dimension or other spectra) and of course the sets of points which do not have local dimension.

The idea for multi-multifractal analysis is to iterate infinitely this process and refine the decompositions. The interesting case is when the dimension spectrum is nondegenerate (if not all the spectra are degenerate and constants). We introduce multi-multifractal analysis for dimension, but notice that the constructions for the other spectra are similar. 
In the nondegenerate case we define a set of Gibbs measures (we omit the measures $\mu_{ \pm \infty}$ since we show that they are uniform on their supports, and in particular $\left.\mu_{1}=\mu\right) M_{0}(\mu)=\left\{\mu_{\beta}\right\}_{\beta \in \mathbb{R}}$ where the singularity sets $C_{\alpha}$ satisfy for $\alpha=F^{\prime}(\beta)$,

$$
\mu_{\beta}\left(C_{\alpha}\right)=1 \quad \text { and } \quad \operatorname{HD}\left(C_{\alpha}\right)=f(\alpha)=\operatorname{HD}\left(\mu_{\beta}\right)=d_{\mu_{\beta}} .
$$

Then multifractal analysis can be represented by the triple $(\mu, F$, $\left.M_{0}(\mu)\right)$.

In fact it is possible to define many infinite sequences of multifractal spectra. Let us describe the second step.

First fix $\beta \in \mathbb{R} \backslash\{1\}$ and realize the multifractal analysis for the measure $\mu_{\beta}$. Define for $(\rho, \tau) \in M_{T}(K) \times \mathbb{R}$,

$$
I_{1}(\rho, \tau)=\frac{h_{\rho}(T)+\tau \int_{\mathbf{K}} \xi_{\beta} d \rho}{\int_{\mathbf{K}} J d \rho} \quad \text { and } \quad F_{1}(\rho, \tau)=\inf _{\rho \in M_{T}(\mathbf{K})}\left(I_{1}(\rho, \tau)\right) \text {. }
$$

We have the following:

- at the first step: $\mu=\mu_{\xi} \longleftrightarrow \beta \in \mathbb{R}, F(\beta)=I\left(\mu_{\beta}, \beta\right) \longleftrightarrow \xi_{\beta}=$ $\beta \xi-F(\beta) J, \mu_{\beta}=\mu_{\xi_{\beta}} \longleftrightarrow f(\alpha)=d_{\mu_{\beta}}$ for $\alpha=F^{\prime}(\beta)$,

- at the second step: $\mu_{\beta}=\mu_{\xi_{\beta}} \longleftrightarrow \tau \in \mathbb{R}, F_{1}(\tau)=I_{1}\left(\mu_{\beta, \tau}, \tau\right) \longleftrightarrow$ $\zeta_{\tau}=\tau \xi_{\beta}-F_{1}(\tau) J, \mu_{\beta, \tau}=\mu_{\zeta_{\tau}} \longleftrightarrow f_{1}(\alpha)=d_{\mu_{\beta, \tau}}=\operatorname{HD}\left(C_{\beta, \alpha}\right)$ for $\alpha=F_{1}^{\prime}(\beta)$ and $C_{\beta, \alpha}=\left\{x \in K: d_{\mu_{\beta}}(x)=\alpha\right\}$. If $M_{1}\left(\mu_{\beta}\right)=\left\{\mu_{\beta, \tau}\right\}_{\tau \in \mathbb{R}}$, we have then defined a new triple $\left(\mu_{\beta}, F_{1}, M_{1}\left(\mu_{\beta}\right)\right)$.

We can iterate this construction step by step at any level.

Suppose that multifractal analysis has been defined at level $n$. We have then for $\left(\beta_{1}, \ldots, \beta_{n-1}\right) \in \mathbb{R}^{n-1}$ a triple

$$
\left(\mu_{\beta_{1}, \ldots, \beta_{n-1}}, F_{\beta_{1}, \ldots, \beta_{n-1}},\left\{\mu_{\beta_{1}, \ldots, \beta_{n-1}, \beta}\right\}_{\beta \in \mathbb{R}}\right)
$$

and

$$
\begin{aligned}
\mu_{\beta_{1}, \ldots, \beta_{n-1}}= & \mu_{\xi_{\beta_{1}, \ldots, \beta_{n-1}}} \\
& \longleftrightarrow \beta_{n} \in \mathbb{R}, F_{\beta_{1}, \ldots, \beta_{n-1}}\left(\beta_{n}\right) \\
& \longleftrightarrow \beta_{n} \in \mathbb{R}, \mu_{\beta_{1}, \ldots, \beta_{n-1}, \beta_{n}}, \mu_{\xi_{\beta_{1}, \ldots, \beta_{n-1}, \beta_{n}}} \\
& \longleftrightarrow f(\alpha)=\operatorname{HD}\left(C_{\beta_{1}, \ldots, \beta_{n-1}, \alpha}\right)=\operatorname{HD}\left(\mu_{\beta_{1}, \ldots, \beta_{n-1}, \beta_{n}}\right),
\end{aligned}
$$


for $\alpha=F_{\beta_{1}, \ldots, \beta_{n-1}}^{\prime}\left(\beta_{n}\right)$ where we have

$$
C_{\beta_{1}, \ldots, \beta_{n-1}, \alpha}=\left\{x: \frac{\ln \mu_{\beta_{1}, \ldots, \beta_{n-1}}(B(x, r))}{\ln r} \underset{r \rightarrow 0}{\longrightarrow} \alpha\right\} .
$$

We have then defined a new triple

$$
\left(\mu_{\beta_{1}, \ldots, \beta_{n-1}, \beta_{n}}, F_{\beta_{1}, \ldots, \beta_{n-1}, \beta_{n}},\left\{\mu_{\beta_{1}, \ldots, \beta_{n-1}, \beta_{n}, \beta}\right\}_{\beta \in \mathbb{R}}\right),
$$

where we omit the two extremal measures $\mu_{\beta_{1}, \ldots, \beta_{n-1}, \beta_{n}, \pm \infty}$.

If at the first level the spectrum is nondegenerate, then it is nondegenerate at any level. We have seen that it is degenerate at the first level if and only if the two potentials $\xi$ and $J$ are cohomologous to constants. Since at any level it is a linear combination of the functions $\xi$ and $J$ it is never degenerate.

Concerning local Lyapunov exponents this is the same behaviour than for dimension. If the multi-multifractal spectrum is nondegenerate at the first step ( $J$ is not cohomologous to a constant), then it is not degenerate at any step.

The behaviour for local entropies is different. For example at the first level it may be degenerate ( $\xi$ is cohomologous to a constant), but at the second level it may be not since for any real number $\beta \neq 1$, $\xi_{\beta}=\beta \xi-F(\beta) J$ is not cohomologous to a constant, and in fact it is not at any further level.

We omit at each step the extremal measures $\mu_{\beta_{1}, \ldots, \beta_{n-1}, \beta_{n}, \pm \infty}$ obtained at the limits when $|\beta|$ goes to $+\infty$. In fact at any level these measures are uniform on their supports and then imply degenerate spectra.

We will see it on a very simple example on the unit interval, namely a linear Markov map modeled by the full shift on 3 symbols.

Let us describe this dynamical system by the following simple model.

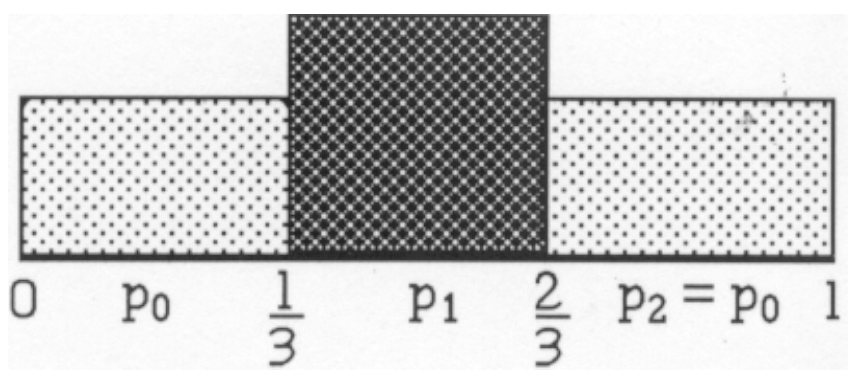

Figure 1. The measure $\mu$ given by $p_{1}+2 p_{0}=1$. 
(for example $p_{0}=0.3$ and $p_{1}=0.4$ ), and

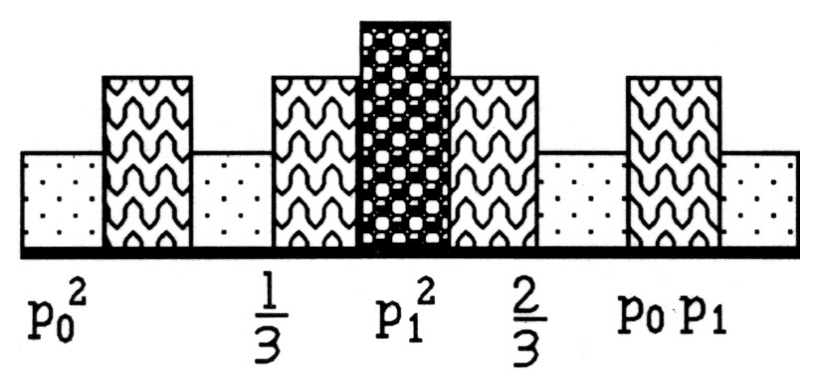

Figure 2. The measure $\mu$ given at the second step (and so on...).

In the computation of the partition functions (7), the different sets $B_{j(\beta, n)}^{n}$ that are selected $(29)\left(=A_{j(\beta, n)}^{n}(27)\right.$ since $J$ is constant: the partitions are uniform, $|V(U)|=3^{-n}$ ) when $\beta \longrightarrow+\infty$, are in fact the intervals where the distribution of the mass $\mu(V(U))$ is the largest. They are actually the central intervals $\left[1 / 2-1 /\left(2 \cdot 3^{n}\right), 1 / 2+1 /\left(2 \cdot 3^{n}\right)\right]$ of measure $p_{1}^{n}$ which covers at the limit the set $\{1 / 2\}$. We have then $\mu_{+\infty}=\delta_{1 / 2}$ and $d_{\mu_{+\infty}}=0$.

When $\beta \longrightarrow-\infty$, it is the set of intervals where the distribution of the mass $\mu(V(U))$ is the smallest. In fact we select the sets

$$
\bigcup_{k=0}^{3^{n-1}-1}\left(\left[\frac{3 k}{3^{n}}, \frac{3 k+1}{3^{n}}\right] \cup\left[\frac{3 k+2}{3^{n}}, \frac{3 k+3}{3^{n}}\right]\right)
$$

composed of $2^{n}$ intervals of measure $p_{0}^{n}$, which cover at the limit the tryadic Cantor set. We obtain therefore that $\mu_{-\infty}$ is the uniform measure on the Cantor set for which the dimension spectrum is degenerate at the point $d_{\mu_{-\infty}}=\ln 2 / \ln 3$.

The multifractal analysis implies the following results.

1) $\operatorname{HD}\left(\left\{x: d_{\mu}(x)\right.\right.$ does not exists $\left.\}\right)=1$. This set contains for example the set of points obtained by iterations of the boundaries: for these special points we have

$$
\underline{d}_{\mu}(x)=\alpha_{+\infty}=-\frac{\ln p_{1}}{\ln 3} \quad \text { and } \quad \bar{d}_{\mu}(x)=\alpha_{-\infty}=-\frac{\ln p_{0}}{\ln 3} .
$$

In higher dimension $n \geq 2$, this set contains iterates of the boundaries of the Markov partition (countable in dimension 1) and then has Hausdorff dimension greater or equal to 1 (equal to $n$ in general). 
2) The dimension spectrum is real analytic on the interval $] \alpha_{+\infty}$, $\alpha_{-\infty}[$.

3) For all $\beta \in \mathbb{R}, \mu_{\beta}$ is exact dimensional and $d_{\mu_{\beta}}=\beta F^{\prime}(\beta)-F(\beta)$ where we have

$$
F(\beta)=-\frac{\ln \left(2 p_{0}^{\beta}+p_{1}^{\beta}\right)}{\ln 3} \quad \text { and } \quad F^{\prime}(\beta)=-\frac{2 p_{0}^{\beta} \ln p_{0}+p_{1}^{\beta} \ln p_{1}}{\left(2 p_{0}^{\beta}+p_{1}^{\beta}\right) \ln 3}
$$

For $\beta=1, \mu_{1}=\mu$ is exact dimensional.

We see that the extremal measures $\mu_{ \pm \infty}$ are uniform measures on their supports. This phenomenon seems to be general, and it is quite clear for linear Markov maps equipped with Gibbs measures. The next step is for subshifts of finite type where things are more complicated (case of the digraph recursive fractals) in the nondegenerate case.

We have seen in (29) that for any real number $\beta$ the set $B_{j(\beta, n)}^{n}$ consists of elements of the Markov partition $\mathcal{P}_{n}$ (of "same" measure $\exp (i(\beta, n))$ and "same" length $\exp (j(\beta, n)) \sim b(\beta, n)^{-n}$ in the order $(1 / n) \ln )$ indicates at the step $n$ the distribution of the mass $\mu(V(U))^{\beta}$ of the partition function (7) and where the large deviations occur (6).

In the order $(1 / n) \ln$ some small variations for the mass of the elements of $B_{j(\beta, n)}^{n}$ occur which imply the multifractality of the measure $\mu_{\beta}$ (multi-multifractality at the second level).

The situation is different for the extremal measures $\mu_{ \pm \infty}$ given by the limits of the measures $\mu_{\beta}$ when $|\beta| \longrightarrow+\infty$.

For the measure $\mu_{-\infty}$ the elements of $\mathcal{P}_{n}$ which cover at the limit the set $\mathbf{K}^{(-\infty)}$ are those which satisfy the following:

$$
0<\mu(V(U))=\min _{V(U) \in \mathcal{P}_{n}} \mu(V(U)) .
$$

In the same way, for the measure $\mu_{+\infty}$ the elements of $\mathcal{P}_{n}$ which cover at the limit the set $\mathbf{K}^{(+\infty)}$ are those which satisfy the following

$$
0<\mu(V(U))=\max _{V(U) \in \mathcal{P}_{n}} \mu(V(U)) .
$$

In our example these sets are respectively the $2^{n}$ intervals of measures $p_{0}^{n}$ and the central intervals of measures $p_{1}^{n}$.

Therefore if we want to realize the multi-multifractality analysis of the measures $\mu_{ \pm \infty}$ at the second level, we get for example $\mu_{-\infty,-\infty}=$ $\mu_{-\infty}$ and $\mu_{+\infty,+\infty}=\mu_{+\infty}$ and finally for any $\tau \in \overline{\mathbb{R}}, \mu_{-\infty, \tau}=\mu_{-\infty}$ and $\mu_{+\infty, \tau}=\mu_{+\infty}$. This gives Theorem D. 
Here we present the different graphs of the functions we have studied for the particular values: $p_{0}=0.3$ and $p_{1}=0.4$ : Figure 3 : the function $F$; Figure 4 : the derivative $F^{\prime}$; Figure 5 : the function which represents the distribution of $\beta \longmapsto d_{\mu_{\beta}}$; (see (41); Figure 6: the dimension spectrum : $\alpha \longmapsto f(\alpha)$.

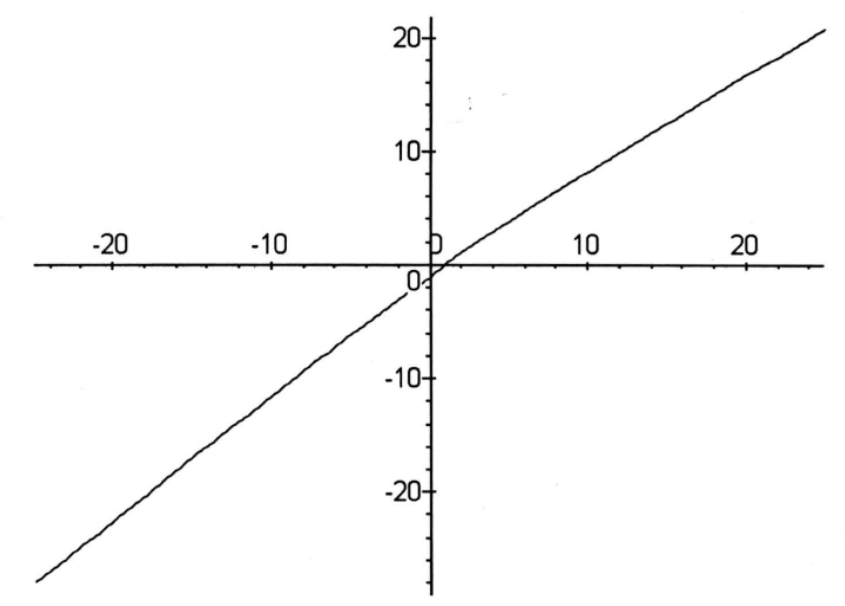

Figure 3. The free energy function $F: \mathbb{R} \longrightarrow \mathbb{R}, \beta \longmapsto F(\beta)$.

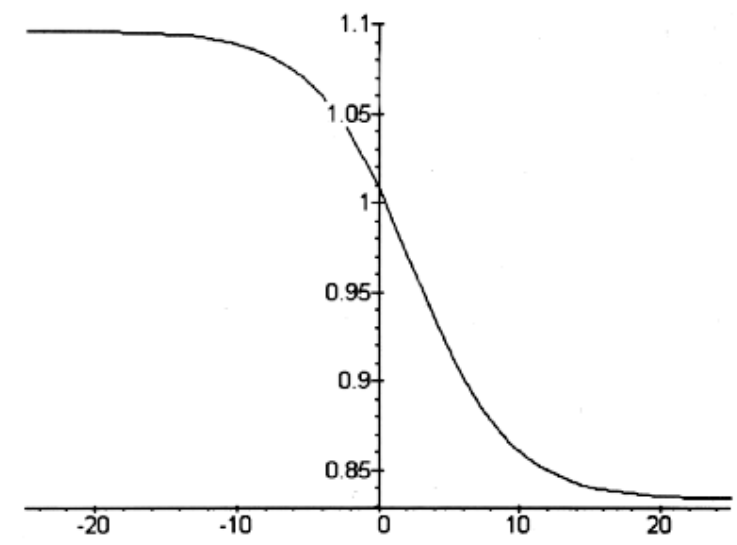

Figure 4. The derivative of the free energy function $\left.F^{\prime}: \mathbb{R} \longrightarrow\right] \alpha_{+\infty}, \alpha_{-\infty}\left[, \beta \longmapsto F^{\prime}(\beta)\right.$. 


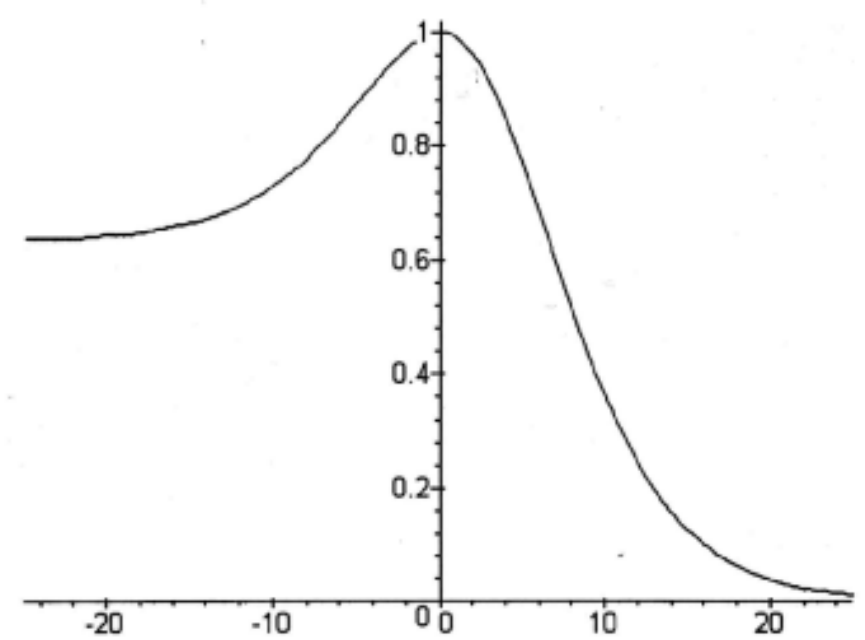

Figure 5. The parametrized dimension spectrum $\left.\left.f_{\beta}: \mathbb{R} \longrightarrow\right] 0,1\right], \beta \longmapsto \beta F^{\prime}(\beta)-F(\beta)$.

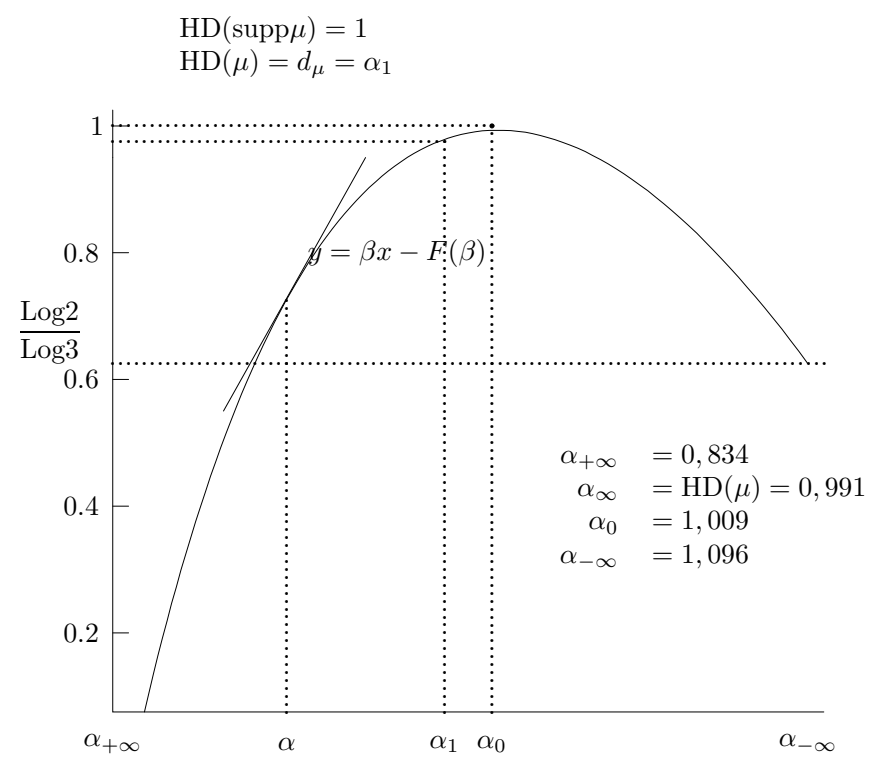

Figure 6. The dimension spectrum $f:\left[\alpha_{+\infty}, \alpha_{-\infty}\right] \longrightarrow[0,1], \alpha \longmapsto f(\alpha)$. 


\section{Discussion and questions.}

We may summarize the differents results concerning the measure $\mu$. The measure $\mu$ is exact dimensional, i.e. $d_{\mu}(x)=h_{\mu} / \chi_{\mu} \mu$ almost everywhere, although we have the following

$$
\operatorname{HD}\left(\left\{x: d_{\mu}(x) \text { does not exist }\right\}\right)=n .
$$

For $\alpha=F^{\prime}(1)=d_{\mu}$, we have $\mu\left(\mathbf{K}^{(\alpha)}\right)=1$ which gives the completness of the measure.

There are limiting constructions for the $\mathbf{K}^{(\alpha)}$ when $\alpha \longrightarrow \alpha_{ \pm \infty}$. The sets $\mathbf{K}^{\left(\alpha_{ \pm \infty}\right)}$ are the supports of the measures $\mu_{ \pm \infty}$ which are uniform on their supports. Therefore their multifractal and multimultifractal are reduced to points

$$
d_{\mu_{-\infty}}=\frac{h_{\mu_{-\infty}}}{\chi_{\mu_{-\infty}}} \quad \text { and } \quad d_{\mu_{+\infty}}=\frac{h_{\mu_{+\infty}}}{\chi_{\mu_{+\infty}}} .
$$

The disjointness conditions on the sets $J(\gamma)$ are those for Markov partitions, i.e. the interiors are disjoints and they intersect at most at their boundaries which are of measure 0 for any Gibbs measure. Like for the example, all the points on the boundaries belong to the set

$$
\left\{x: d_{\mu}(x) \text { does not exist }\right\}
$$

which is not countable in dimension geater or equal to 2 .

If the graph is not strongly connected, we analyse all the strongly connected components of the graph, i.e. if the matrix $A$ (see Section 3.1 ) is not reducible, we decompose it into irreducible components.

To each irreducible component $A_{1 \leq j \leq p}^{(j)}$ we associate in the same fashion as in the digraph recursive fractal sets the singularity sets and the different dimension spectra which may or not intersect with the others. For any value $\alpha \in\left[\alpha_{\min }, \alpha_{\max }\right]$, there are at most $p$ different singularity sets where $C_{\alpha}^{(j)}=\left\{x: d_{\mu}(x)=\alpha\right\}$ (which may be $\left.=\varnothing\right)$, and therefore we define

$$
\begin{aligned}
f(\alpha) & =\max _{1 \leq j \leq p} \operatorname{HD}\left(C_{\alpha}^{(j)}\right) \quad(\equiv-\infty \text { if all the singularity sets are } \varnothing) \\
& =\max _{1 \leq j \leq p} f^{(j)}(\alpha)
\end{aligned}
$$

where $f^{(j)}$ is the dimension spectrum of the measure $\mu$ restricted to the set generated by the $\mathrm{j}$-th strongly connected component. 
The result means that we get for any positive real number $\alpha, f(\alpha)$ to be the greatest Hausdorff dimension of the singularity sets $C_{\alpha}^{(j)}$ (since we have the following: $\operatorname{HD}(E \cup F)=\max \{\operatorname{HD}(E), \operatorname{HD}(F)\})$.

We have seen in (43) in the nondegenerate case that $F^{\prime \prime}<0$ (if and only if the Hölder continuous functions $\xi$ and $J$ are not cohomologous to constants), and we get finally that $f^{\prime \prime}<0$ on $] \alpha_{\min }, \alpha_{\max }[$ since we have $f^{\prime \prime}(\alpha)=1 / F^{\prime \prime}(\beta)$. Then we have for any real number $\beta \in \mathbb{R}$ and $\alpha \in] \alpha_{\min }, \alpha_{\max }\left[, F^{\prime \prime}(\beta)<0\right.$ and $f^{\prime \prime}(\alpha)<0$, and the value 0 is never achieved.

The challenging question at this moment comes from the concept of rigidity and the conjecture that the dimension spectrum is an invariant for dynamical systems modeled by subshifts of finite type.

Rigidity deals with an important problem which is to know if we can restore the dynamics of a dynamical system by recovering information from the different spectra. The aim is to obtain a physical classification of dynamical systems given by maps and Gibbs measures.

Let $(X, \mu, T)$ and $(Y, \rho, S)$ be two topologically equivalent dynamical systems, i.e. there exists a homeomorphism $h: X \longrightarrow Y$. The problem is to know if some of their multifractal spectra coincide then they are smoothly equivalent and $h$ is a diffeomorphism. If there exists a topological conjugacy $\zeta$ between $T$ and $S$, we want to find in all the class of conjugacies a homeomorphism $\phi$ preserving the differentiable structure, $T=S \circ \phi$, and also measure preserving, $\mu=\rho \circ \phi$.

This has been proved in [BPS2] in a very particular case, namely one-dimensional (and two-dimensional) linear Markov maps of $[0,1]$ (or $[0,1]^{2}$ ) modeled by the full shift on two symbols (where all the things work). We believe that this assertion is true for linear Markov maps of the unit interval (or $[0,1]^{2}$ ) modeled by the full shift on $p \geq 2$ symbols. The generalization of this statement will be for arbitrary subshifts of finite type $\Sigma_{A}^{+}$.

We believe that multifractal dimension spectrum is only needed to recover information, but if necessary one can use multi-multifractal analysis.

\section{References.}

[AP] Arbeiter, M., Patzschke, N., Random self-similar multifractals. Mat. Nachr. 181 (1996), 5-42. 
[BPS1] Barreira, A., Pesin, Y., Schmeling, J., Dimension and product structure of hyperbolic measures. Ann. of Math. 149 (1999), 755-783.

[BPS2] Barreira, A., Pesin, Y., Schmeling, J., On a general concept of multifractality: multifractal for dimensions, entropies, and Lyapunov exponents. Multifractal rigidity. Chaos 7 (1997), 27-38.

[Bo] Bowen, R., Equilibrium states and the ergodic theory of Anosoff diffeomorphisms. Lecture Notes in Math. 470 (1973). Springer.

[BMP] Brown, G., Michon G., Peyrière, J., On the multifractal analysis of measures. J. Stat. Phys. 66 (1992), 775-790.

[CM] Cawley, E., Mauldin, R. D., Multifractal decomposition of Moran fractals. Advances in Math. 92 (1992), 196-236.

[CLP] Collet, P., Lebowitz, J. L., Porzio, A., Dimension spectrum of some dynamical systems. J. Stat. Phys. 47 (1987), 609-644.

[C] Cutler, C. D., Connecting ergodicity and dimension in dynamical systems. Ergodic Theory \& Dynamical Systems 10 (1990), 451-462.

[DGS] Denker, M., Grillenberger, C., Sigmund, K., Ergodicity theory on compact spaces. Lecture Notes in Math. 527 (1976). Springer.

[EM] Edgar, G. A., Mauldin, R. D., Multifractal decomposition of digraph recursive fractals. Proc. London. Math. Soc. 65 (1992), 604-628.

[E] Ellis, R. S., Entropy, large deviations and statistical mechanics. Grundlehren der Mathematischen Wissenschaften 271 (1985). Springer.

[F1] Falconer, K. J., The multifractal spectrum of statistically self-similar measures. J. Theor. Probab. 3 (1994), 681-702.

[F2] Falconer, K. J., Techniques in fractal geometry. J. Wiley \& Sons, 1997.

[GHP] Grassberger, P., Hentschel, H. G. E., Procaccia, I., On the characterization of chaotic motions. Lect. Notes Phys. 179 (1983), 212-221.

[HJKPS] Hasley, T. C., Jensen, M. H., Kadanoff, L. P., Procaccia, I., Shreiman, B. I., Fractal measures and their singularities: the characterization of strange sets. Phys. Rev. A 33 (1986), 1141-1151.

[HP] Hentschel, H. G. E., Procaccia, I., The infinite number of generalized dimensions of fractals and strange attractors. Physica D 9 (1983), 435444.

[HW] Holley, R., Waymire, E., Multifractal dimensions and scaling exponents for strongly bounded random cascades. Ann. App. Probab. 4 (1992), 819-845.

[HY] Hu, H., Young, L.-S., Nonexistence of SBR measures for some diffeomorphisms that are "almost Anosov". Ergodic Theory \& Dynamical Systems 15 (1995), 67-76.

[K] King, J., The singularity spectrum for general Sierpinski carpets. Advances in Math. 116 (1995), 1-11. 
[L1] Ledrappier, F., Some relations between dimension and Lyapunov exponents. Comm. Math. Phys. 81 (1981), 229-238.

[L2] Ledrappier, F., On the dimension of some graphs. Contem. Math. 135 (1992), 285-293.

[LM] Ledrappier, F., Misiurewicz, M., Dimension of invariant measures for maps with exponent zero. Ergodic Theory \& Dynamical Systems $\mathbf{5}$ (1985), 545-556.

[LM] Ledrappier, F., Young, L.-S., The metric entropy of diffeomorphisms. Part II: Relations between entropy, exponents and dimension. Ann. of Math. 122 (1985), 540-574.

[Lo] Lopes, A. O., The dimension spectrum of the maximal measure. SIAM J. Math. Anal. 20 (1989), 1243-1254.

[M] Mañé, R., The Hausdorff dimension of horseshoes of diffeomorphisms of surfaces. Bol. Soc. Bras. Mat. 20 (1990), 1-24.

[Ma] Manning, A., A relation between Lyapunov exponents, Hausdorff dimension and entropy. Ergodic Theory \& Dynamical Systems 1 (1981), 451-459.

[MC] Manning, A., Mc Cluskey, H., Hausdorff dimensions for horseshoes. Ergodic Theory \& Dynamical Systems 5 (1983), 251-260.

[Mo] Moran, M., Hausdorff measure of infinitely generated self-similar sets. Monastsh. Math. 4 (1996), 387-399.

[MR] Moran, M., Rey, J.-M., Geometry of self-similar measures. Acad. Sci. Fennicae Math. 2 (1997), 365-386.

[O1] Olsen, L., A multifractal formalism. Advances in Math. 116 (1995), 82-196.

[O2] Olsen, L., Self-affine multifractal Sierpinski sponges in $\mathbb{R}^{n}$. Pacific J. Math. 183 (1998), 143-199.

[P1] Pesin, Y., On rigorous mathematical definitions of correlation dimension and generalized spectrum for dimensions. J. Stat. Phys. 71 (1993), 529547.

[P2] Pesin, Y., Dimension theory in dynamical systems: rigorous results and applications. Chicago Lectures in Mathematics. The University Chicago Press, 1997.

[PT] Pesin, Y., Tempelman, A., Correlation of measures invariant under group actions. Rand. \& Comp. Dyn. 3 (1995), 137-156.

[R] Rand, D. A., The singularity spectrum $f(\alpha)$ for cookie-cutters. Ergodic Theory \& Dynamical Systems 9 (1989), 527-541.

[Ri] Riedi, R., An improved multifractal formalism and self-similar measures. J. Math. Anal. Appl. 189 (1995), 462-490.

[Ru] Ruelle, D., Thermodynamic Formalism. Addison-Wesley, 1978. 
[S] Shereshevski, M. A., A complement to Young's theorem on measure dimension: the difference between lower and upper pointwise dimensions. Nonlinearity 4 (1991), 15-25.

[Si1] Simpelaere, D., Dimension spectrum of Axiom A diffeomorphisms. I: The Bowen-Margulis measure. II: Gibbs measures. J. Stat. Phys. 76 (1994), 1329-1375.

[Si2] Simpelaere, D., Mean of the singularities of a Gibbs measure. Comm. Math. Phys. 179 (1996), 489-510.

[Y] Young, L.-S., Dimension, entropy and Lyapunov exponents. Ergodic Theory \& Dynamical Systems 2 (1982), 109-124.

Recibido: 11 de mayo de 1.999

Revisado: 29 de noviembre de 1.999

Dominique Simpelaere

Université Paris 6

Laboratoire de Probabilités

4, Place Jussieu, tour 56

75252 Paris cedex 05, FRANCE

ds@ccr.jussieu.fr

and

Université Paris 12

61, Avenue du Général de Gaulle

94010 Créteil cedex, FRANCE

simpelaere@univ-paris12.fr 